\title{
An Extended Grid of Nova Models: II. The Parameter Space of Nova Outbursts
}

\author{
O. Yaron, ${ }^{1}$ D. Prialnik, ${ }^{1}$ M. M. Shara ${ }^{2}$ and A. Kovetz ${ }^{1,3}$
}

\begin{abstract}
This paper is a sequel to an earlier paper devoted to multiple, multicycle nova evolution models (Prialnik \& Kovetz (1995), first paper of the series), which showed that the different characteristics of nova outbursts can be reproduced by varying the values of three basic and independent parameters: the white dwarf mass- $M_{W D}$, the temperature of its isothermal core- $T_{W D}$ and the mass transfer rate- $\dot{M}$. Here we show that the parameter space is constrained by several analytical considerations and find its limiting surfaces. Consequently, we extend the grid of multicycle nova evolution models presented in Paper I to its limits, adding multicycle nova outburst calculations for a considerable number of new parameter combinations. In particular, the extended parameter space that produces nova eruptions includes low mass transfer rates down to $5 \times 10^{-13} M_{\odot} \mathrm{yr}^{-1}$, and more models for low $T_{W D}$. Resulting characteristics of these runs are added to the former parameter combination results, to provide a full grid spanning the entire parameter space for Carbon-Oxygen white dwarfs. The full grid covers the entire range of observed nova characteristics, even those of peculiar objects, which have not been numerically reproduced until now. Most remarkably, runs for very low $\dot{M}$ lead to very high values for some characteristics, such as outburst amplitude $A \gtrsim 20$, high super-Eddington luminosities at maximum, heavy element abundance of the ejecta $Z_{e j} \approx 0.63$ and high ejected masses $m_{e j} \approx 7 \times 10^{-4} M_{\odot}$.
\end{abstract}

Subject headings: accretion, accretion disks — binaries: close — novae, cataclysmic variables — white dwarfs

\footnotetext{
${ }^{1}$ Department of Geophysics and Planetary Sciences, Sackler Faculty of Exact Sciences, Tel Aviv University, Ramat Aviv 69978, Israel

${ }^{2}$ Department of Astrophysics, American Museum of Natural History, Central Park West and 79th street, New York, NY 10024-5192

${ }^{3}$ School of Physics and Astronomy, Sackler Faculty of Exact Sciences, Tel Aviv University, Ramat Aviv 69978, Israel
} 


\section{INTRODUCTION}

The realization that Classical Novae $(\mathrm{CN})$ are due to thermonuclear runaways (TNR) on the surfaces of white dwarf (WD) stars in close, mass-transferring binary systems (Starrfield, Sparks \& Truran 1972) is now three decades behind us. Direct observations of novae (summarized in e.g. Payne-Gaposchkin (1957)) had already demonstrated that large ranges in ejecta velocity and metallicity occur in novae, and later interpretations of the data suggested that ejecta masses varied strongly from object to object. The first simulations by nova theorists, trying to make sense of these observations, involved much groping into various corners of parameter space. Early triumphs included an explanation of the UV emission and high bolometric luminosities of novae after eruption (Starrfield, Sparks \& Truran 1976); fast and moderately slow nova models (Prialnik, Shara \& Shaviv 1978); natural enrichment of ejecta in heavy elements via diffusion (Prialnik \& Kovetz 1985) and via mixing from the underlying white dwarf (MacDonald 1983; Kutter \& Sparks 1987); the realization that nuclear burning with EUV emission could occur without mass ejection (perhaps leading to SNIa or super soft X-ray sources) (Shara, Prialnik \& Shaviv 1977) and novae recurring on timescales as short as a few years (Starrfield, Sparks \& Shaviv 1987).

The first decade of nova simulations crystallized the understanding that a space of three independent parameters controls the behavior of a classical nova eruption: the white dwarf mass, the white dwarf temperature (or luminosity) and the mass accretion rate from the companion (Shara, Prialnik \& Shaviv 1980; Prialnik 1995). Increased computer power, and codes including better physics lead to an ever-increasingly sophisticated series of eruption simulations. Once multi-cycle simulations became possible, the arbitrariness of initial conditions could be eliminated, and systematic surveys throughout the three-dimensional space became a real possibility. These culminated in an extended grid of multicycle nova evolution models (Prialnik \& Kovetz (1995), hereafter Paper I) which have been extensively used by researchers.

While Prialnik \& Kovetz covered virtually all of the $\left(M_{W D}, T_{W D}, \dot{M}\right)$ space then thought likely to be reached by novae, more recent work (Hurley \& Shara 2003) has shown that cataclysmic variables are likely to evolve considerably beyond the boundaries of their calculations. Remarkably, we find (in the present study) that some of these systems (parameter combinations) still produce nova eruptions. Amongst these are models with extreme values of $M_{W D}$, $T_{W D}$ and $\dot{M}$ which can give rise to "extreme novae" - objects with eruption luminosities, metallicities, and/or ejecta masses and velocities significantly larger or significantly smaller than any yet predicted by simulations.

The existence of very unusual eruptive systems like the "Red Variable" in M31 (Mould 2000) or V838 Mon (Bond et al. 2003) are already sufficient justification for extending nova 
simulation grids to cover all conceivable cases. Each of these unusual variables has properties (particularly luminosity) reminiscent of novae, and we will examine possible connections in a future paper. But of equal interest is the physical insight that these new simulations can provide, as important input in trying to determine the very long term evolution of cataclysmic binaries, and relationships between the various subclasses of CVs. Most surprising of all is the non-linear behavior of some of the most extreme new nova models. Simple extrapolations from previous simulations give answers that may be wrong by large factors. This is because dominance of competing timescales and physical effects changes with movement through the three-dimensional nova parameter space.

It is perhaps not too surprising that $\dot{M}$ lower than $10^{-11} M_{\odot} \mathrm{yr}^{-1}$ (the lowest values considered in Paper I) can occur, especially as a mass-accreting WD whittles down its donor companion to the brown dwarf mass range. Equally understandable is that WD core temperatures can drop to or below $10^{7} \mathrm{~K}$, for WDs accreting at very low rates for many Gyr, whereas Paper I considered only hot WDs accreting at the lowest rate. Both these effects low mass accretion rate and cold WDs - are extensively studied in the present work. What is less intuitively obvious is that CO WDs with masses well under the $0.65 M_{\odot}$ (the lower limit of the Paper I study) are also expected. Mass stripping via common envelope evolution can produce a naked helium star, which evolves to the helium giant branch and then becomes a low-mass CO WD, as illustrated by the example of a long-term binary evolution track summarized in Table 1 (Hurley, private communication). The existence of low-mass $\left(\lesssim 0.50 M_{\odot}\right)$ WDs resulting from close binary evolution was also discussed by Marsh et al. (1995) and Althaus et al. (2004) (and references therein). The behavior of such low-mass CO WDs accreting hydrogen-rich material is also examined, for the first time, in the present work.

The methods of numerical computation, the grid of results, and the range of nova parameter space covered are discussed in $\S 2$. In $\S 3$ we discuss the existence of a confined region of the three-dimensional parameter space within which conditions for nova outbursts are satisfied. A brief summary and conclusions are given in $\S 4$.

\section{NUMERICAL COMPUTATIONS}

The hydrodynamic Lagrangian stellar evolution code by which the present study was performed is the code presented in Paper I. It includes OPAL opacities, an extended nuclear reactions network comprising of 40 heavy element isotopes and a mass-loss algorithm that applies a steady, optically thick supersonic wind solution (following the phase of rapid expansion). In addition, diffusion is computed for all elements, accretional heating is taken into 
account and convective fluxes are calculated according to the mixing length theory. Initial models were prepared for four WD-mass values and three temperatures by cooling WD models from higher temperatures. Each nova model was followed through several consecutive outburst cycles in order to eliminate the effect of the initial configuration. One typical cycle was then chosen as representative of each parameter combination.

The original grid presented in Paper I consisted of 64 parameter combinations: four $M_{W D}$ values - $0.65,1.00,1.25 \& 1.40 M_{\odot}$, three $T_{W D}$ values- $10,30 \& 50 \times 10^{6} \mathrm{~K}$ and five $\dot{M}$ values $-10^{-6}$ through $10^{-10} M_{\odot} \mathrm{yr}^{-1}$, and four calculations for the highest temperature value with $\dot{M}$ of $10^{-11} M_{\odot} \mathrm{yr}^{-1}$. In the present work we have extended the grid to lower accretion rates, down to $\dot{M}=5 \times 10^{-13} M_{\odot} \mathrm{yr}^{-1}$ for all values of WD mass and temperature and we have added calculations for $M_{W D}=0.4 M_{\odot}$, amounting to about 30 new parameter combinations in all.

Tables $2 \& 3$ list the results of our complete grid of parameter combinations. The tables are presented in a manner similar to that of Paper I: Table 2 lists properties that are related to the accretion phase and the onset of the outburst, whereas Table 3 presents characteristics of the outburst itself. The properties displayed in Table 2 are: the accreted and ejected masses $m_{a c c}, m_{e j}$; the helium mass fraction of the envelope and the ejecta $Y_{e n v}, Y_{e j}$; the heavy element mass fractions $Z_{e n v}, Z_{e j}$; and the maximum temperature attained in the burning shell $T_{\max }$. The characteristics displayed in Table 3 are: the maximal expansion velocity $v_{\max }$, its average over the whole mass-loss phase $v_{a v}$, the maximal luminosity attained during the outburst $L_{4, \max }$, the amplitude of the outburst in magnitudes and three typical timescalesthe time of decline of bolometric luminosity by 3 mag. $t_{3, b o l}$, the duration of the mass-loss phase $t_{m-l}$, and the recurrence period of the outbursts $P_{r e c} \equiv m_{a c c} / \dot{M}$.

Some parameter combinations yield peculiar results, such as extremely high (highly super-Eddington) luminosities, which will require further investigation; for a few runs the determination of timescales, such as $t_{3, b o l}$ and $t_{m-l}$ may not be accurate, due to fluctuations of the light curve. For example, the combination 125.30.13 (standing for $1.25 M_{\odot} \mathrm{WD}$, with $T_{W D}=30 \times 10^{6} \mathrm{~K}$ and $\dot{M}=10^{-13} M_{\odot} \mathrm{yr}^{-1}$ ) produces a sharp quick peak at outburst, resulting in very small $t_{3, b o l}$ and $t_{m-l}$ (Table 3 ), followed by a second increase in luminosity and temperature, but one that does not eject more material. The values stated in the table relate only to the first quick rise.

One of the most important characteristics of a classical nova outburst is the time of decline of the visual magnitude, which serves as a basis for the "speed class" classification of novae and on which the role of novae as standard candles is based. In Fig. 1 we show the distribution of the time of decline $t_{3, v i s}$ (which is of the order of $t_{m-l}$ as presented in Table 3) versus the outburst amplitude $A$ for 79 observed galactic novae from the Duerbeck 
catalog (Duerbeck 1987), together with results of the sub-set of 75 models that produced nova outbursts. Three points should be noted. First, the general tendency of decreasing $t_{3}$ for increasing $A$ is clearly seen. Secondly, the model results provide full coverage of observations in the $\left[A, t_{3}\right]$ plane. In this respect, it should be noted that the density of points on the plane has no significance, since the calculated models represent parameter combinations (they are not based on a population synthesis model). Finally, we predict the existence of a class of low amplitude novae (with $A \sim 7$ ) with decline times ranging from 7 days to over a year. We suggest that these objects have either not yet been observed, or remain characterized as variables of some other type.

The effect of each of the three input parameters on the resulting nova characteristic was already discussed in Paper I and also by Schwartzman et al. (1994). The outcome is not always self-evident and the trends are not necessarily monotonic. To illustrate the behavior of some of the more interesting characteristics, we plot in Fig. 2 the model results over the $\left(M_{W D}, \dot{M}\right)$ plane for a given $T_{W D}$ value. Panel 2 a displays the maximum luminosity attained $L_{4, \max }$ together with the $L_{E d d}$ surface, enabling the identification of super- and sub-Eddington luminosities. The lowest sub-Eddington luminosities are obtained for the low $M_{W D}$, high $\dot{M}$ corner, identified as the region of least-powerful nova outbursts. Panel $2 \mathrm{~b}$ displays the ejected mass $m_{e j}$ (on a logarithmic scale). The highest ejected masses are obtained for the lowest WD masses through the entire range of accretion rates. This is because the accreted masses necessary to trigger a TNR are higher for lower mass WDs (see $\S 3$ ) whereas at the same time, the weaker gravitational potential enables more massive ejecta, despite the typically lower outburst intensity. In panel $2 \mathrm{c}$ we show the maximum expansion velocities $v_{\max }$, which exhibit a similar behavior to that of the maximum luminosity surface. While the low $M_{W D}$, high $\dot{M}$ domain is that of weakest novae, the high $M_{W D}$, low $\dot{M}$ corner can be identified as the domain of the most powerful outbursts. The time of bolometric decline, as shown in panel $2 \mathrm{~d}$ (the trend being very similar to that of $t_{m-l}$, which is not displayed here), clearly exhibits, in conjunction with figures $2 \mathrm{a}$ and $2 \mathrm{c}$, the correlation between decline time (speed class) and outburst intensity. The slowest novae (longest decline times) occupy the domain of weakest outbursts and vice versa.

In Table 4 the range of variation of some of the main nova properties is displayed, according to the new extended grid of results (for WD-mass range of $0.65-1.00 M_{\odot}$ ). The parameter combination for which each maximum and minimum value was obtained is indicated next to the value. We note that most of the maximum values result from the new, lower $\dot{M}$, runs.

In Table 5 we present observed ranges of several nova characteristics (cf. Prialnik 1995) together with the ranges obtained from the full grid, for comparison. The ranges resulting 
from model calculations overlap and completely cover the observed ranges.

\section{THE CONSTRAINED PARAMETER SPACE}

As the extension of the three-dimensional parameter space for novae has resulted in the emergence of new features, the question arises: where do we stop, if at all, extending each one of the parameters' ranges? In other words, is the parameter space limited ? We examine this question based on analytical considerations, verified by the results of numerical modeling.

\subsection{Heating versus cooling}

The characteristic timescale for cooling of a WD, $\tau_{\text {cool }}$, is basically a function only of the WD temperature (Mestel 1952). On the other hand, the accretion timescale $\tau_{a c c}$ is directly determined by the accretion rate and indirectly affected by the other parameters through their influence on the mass required to ignite hydrogen, $\tau_{a c c}=m_{a c c}\left(M_{W D}, T_{W D}, \dot{M}\right) / \dot{M}$. A nova outburst can take place only if $\tau_{c o o l}>\tau_{a c c}$; otherwise the temperature cannot rise to

the point of thermonuclear instability. A limiting surface is thus obtained by equating these timescales:

$$
\tau_{\text {cool }}\left(T_{W D}\right)-m_{a c c}\left(M_{W D}, T_{W D}, \dot{M}\right) / \dot{M}=0
$$

Analytically, following Mestel (loc. cit.), if we use the Kramers opacity law for the WD atmosphere, the cooling timescale of a CO WD may be approximated by

$$
\tau_{\text {cool }} \approx 2.5 \times 10^{6}\left(\frac{M / M_{\odot}}{L / L_{\odot}}\right)^{5 / 7} \mathrm{yr},
$$

while the core temperature is approximated by

$$
T_{W D} \approx 4 \times 10^{7}\left(\frac{L / L_{\odot}}{M / M_{\odot}}\right)^{2 / 7} \mathrm{~K} .
$$

On this approximation, then,

$$
\tau_{\text {cool }}\left(T_{W D}\right) \approx 8 \times 10^{7}\left(\frac{10^{7} \mathrm{~K}}{T_{W D}}\right)^{2.5} \mathrm{yr} .
$$

An approximation to the accreted mass can be derived from the requirement that the pressure at the base of the envelope

$$
P_{b}=\frac{G M_{W D} m_{a c c}}{4 \pi R_{W D}^{4}\left(M_{W D}\right)}
$$


exceed a critical prescribed value, say, $P_{\text {crit }} \approx 10^{19} \mathrm{dyn} \mathrm{cm}^{-2}$, in order to obtain a TNR (Fujimoto 1982). Fig. 3, based on grid results, presents yet another numerical validation of

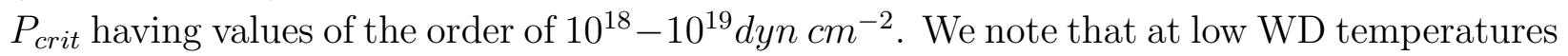
the range of $P_{\text {crit }}$ values is wider, the critical pressure changing with both $\dot{M}$ and $M_{W D}$. Finally, using the Nauenberg approximation (Nauenberg 1972) to the Hamada-Salpeter WD mass-radius relation (Hamada \& Salpeter 1961),

$$
\frac{R_{W D}}{R_{\odot}} \approx 1.12 \times 10^{-2}\left[\left(\frac{M_{W D}}{M_{C h}}\right)^{-2 / 3}-\left(\frac{M_{W D}}{M_{C h}}\right)^{2 / 3}\right]^{1 / 2}
$$

we obtain $m_{a c c}=m_{a c c}\left(M_{W D}\right)$. Substitution of the analytic relations into Eq. 1 yields the desired surface.

A more accurate result is obtained numerically. First, we calculate cooling curves $T_{W D}(t)$ for all the WD masses of the grid (indeed, they are almost independent of $M_{W D}$ ). We define the cooling timescale at any point by $\tau_{\text {cool }}=(d \ln T / d t)^{-1}$ and calculate it for the $T_{W D}$ values of the grid. Then, for each pair $\left(T_{W D}, \dot{M}\right)$, we solve $m_{a c c}\left(M_{W D}, T_{W D}, \dot{M}\right)=\dot{M} \tau_{c o o l}\left(T_{W D}\right)$ to obtain $M_{W D}$, by interpolation on the grid results. We thus obtain the points that satisfy Eq. 1, defining a surface within the parameter space. It is the bottom surface shown in Fig. 4. The surface exhibits a monotonic decline in $M_{W D}$ for each $T_{W D}$, from lower to higher $\dot{M}$, but also some decline of $M_{W D}$ at the same accretion rate from higher to lower $T_{W D}$. As mentioned earlier, the cooling timescales increase with decreasing temperatures. Because longer $\tau_{\text {cool }}$ (relative to $\tau_{a c c}$ ) is what we require for a nova outburst to develop, the WD mass restriction is weaker for lower temperatures. In principle, if $m_{a c c}$ were mainly a function of $M_{W D}$, the condition for a nova outburst to occur would be eventually satisfied for any WD and accretion rate, because $\tau_{\text {cool }}$ would increase, while $\tau_{a c c}$ would remain fixed. However, calculations show that as $T_{W D}$ decreases, $m_{a c c}$ becomes ever more strongly dependent on $T_{W D}$, increasing steeply as the WD cools. Thus, below a certain value of $T_{W D}$, the relation $\tau_{\text {cool }}<\tau_{\text {acc }}$ is maintained while both timescales increase (cf. Schwartzman et al. (1994)). In other words, when mass accumulates slowly as the WD cools down, the accreted mass remains below the critical value while both increase with time.

The cooling time constraint is clearly illustrated in Fig. 5, where examples are shown of the evolution of two characteristics: the maximum temperature within the burning envelope $T_{\text {max }}$ and the total luminosity $L$, for two parameter combinations. A range of accretion rates is displayed, following the evolution over a period of one full cycle, from the beginning of accretion through outburst and decline. The lowest accretion rate for which a "true" massejecting nova is obtained is $\dot{M}=5 \times 10^{-13} M_{\odot} \mathrm{yr}^{-1}$ (and even then, not for all parameter combinations, as seen in the results grid). We see in Fig. 5 that for a slightly lower accretion rate value $2.5 \times 10^{-13} M_{\odot} \mathrm{yr}^{-1}$, the WD cools down faster than it is able to accrete sufficient 
mass to reach TNR conditions. This is clearly seen in the $T_{\max }(t)$ plot: the curve gradually declines, without having reached an outburst. Equally, the luminosity curve remains flat for the lowest $\dot{M}$. The curves for the higher accretion rates exhibit outburst events where $T_{\max }(t)$ exceeds $10^{8} \mathrm{~K}$ and the bolometric luminosity increases above $4-5 \times 10^{4} L_{\odot}$. Note also the obvious but nicely demonstrated fact that as we move from lower to higher accretion rates, the outburst occurs earlier on the timeline, hence the recurrence period becomes shorter.

\subsection{Nuclear versus gravitational energy}

The source of energy for classical nova outbursts is nuclear energy released during the TNR, by burning a fraction $f$ of the hydrogen content of the accreted mass. Thus

$$
E_{n u c}=f X m_{a c c} Q
$$

where: $X \approx 0.7$ is the hydrogen mass fraction in the outer layers of the nova companion star, and $Q \approx 6 \times 10^{18} \mathrm{erg}^{-1}$ is the energy released per gram of burnt hydrogen.

The greatest part of the energy released at outburst is used in lifting the ejected shell from the gravitational potential well of the white dwarf. This gravitational energy may be roughly approximated by

$$
E_{\text {grav }}=\frac{G M_{W D} m_{e j}}{R_{W D}} .
$$

Obviously, a mass ejecting outburst can take place only if $E_{n u c}>E_{\text {grav }}$. Therefore, a limiting surface may be defined by requiring $E_{n u c}=E_{\text {grav }}$,

$$
\frac{m_{a c c}\left(M_{W D}, \dot{M}, T_{W D}\right)}{m_{e j}\left(M_{W D}, \dot{M}, T_{W D}\right)}-\frac{G}{f X Q} \frac{M_{W D}}{R_{W D}}=0 .
$$

As a rough analytical estimate, the difference between $m_{a c c}$ and $m_{e j}$, may be neglected, in which case Eq.(9) simply imposes an upper limit on $M_{W D}$ for a given value of $f$ (e.g., $1.22 M_{\odot}$ for $f=0.1$ and $1.42 M_{\odot}$ for $f=0.3$ ), a flat surface parallel to the $\left[T_{W D}, \dot{M}\right]$ plane in the parameter space. This is already a more severe constraint than just the Chandrasekhar mass limit. Using the results of the numerical computations and taking $f=0.3$ for illustration, we obtain a more significant constraint in the form of a slightly curved surface, the top surface in Fig. 4.

\subsection{Accretion versus Eddington luminosity}

For the nova progenitor to be able to accrete material during the quiescence phase, the accretion luminosity $L_{a c c}$ must be lower than the Eddington critical luminosity $L_{E d d}$. 
Otherwise, radiation pressure would push away and dissipate the accreted material. In fact, the total (net) luminosity of the accreting star should be lower than the Eddington limit, but in most cases the WD intrinsic luminosity is negligible compared with $L_{a c c}$. The accretion luminosity is given by

$$
L_{a c c}=\alpha G M_{W D} \dot{M} / R_{W D}
$$

where $\alpha \sim 0.15$, taking into account accretional heating (Regev \& Shara 1989), and again, using Eq. 6 for $R_{W D}\left(M_{W D}\right)$. Thus a third limiting surface, obtained by equating $L_{a c c}$ and $L_{E d d}$

$$
\dot{M}-\frac{4 \pi c}{\alpha \kappa_{s}} R_{W D}\left(M_{W D}\right)=0
$$

(where $\kappa_{s}$ is the electron scattering opacity coefficient) is shown in Fig. 4 . We note that the result, which in this case relies solely on analytical considerations, is independent of the WD temperature, although it might be indirectly affected by it to some extent through a more realistic opacity coefficient. It is not surprising that the allowed accretion rates decrease with increasing $M_{W D}$.

\subsection{Degeneracy and the WD core temperature}

According to Fig. 4, the relevant region in parameter space where nova eruptions can occur is tube-shaped. Our calculations assumed that the relevant range of WD core temperatures is $10-50 \times 10^{6} \mathrm{~K}$, taking the discrete representative values of 10,30 , and $50 \times 10^{6} \mathrm{~K}$. In fact, the WD temperature is restricted from above and below, as we shall now show.

We may obtain a rough estimate for the upper limit on $T_{W D}$ by setting the Fermi parameter $\varepsilon_{f} \propto(\ln P-2.5 \ln T)$ to zero at the base of the accreted layer, where the pressure, approximately given by Eq. 5 , is of the order of $10^{19} \mathrm{dyn} \mathrm{cm}^{-2}$. Thus assuming that $T_{W D} \approx$ $T_{b}$, we have

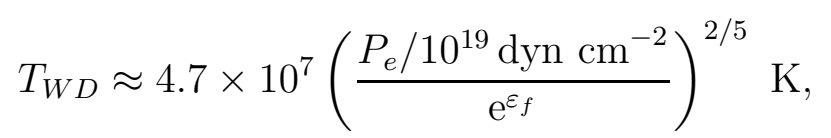

where $P_{e}$ is the electron pressure. Hence if the condition of strong electron degeneracy is expressed as $\varepsilon_{f} \gtrsim 0$, it results in the requirement $T_{W D} \lesssim 5 \times 10^{7} \mathrm{~K}$. Therefore the tube of Fig. 4 has a high temperature end at a $T_{W D}$ of about 50 million degrees. Does it have a low temperature end?

A lower limit on $T_{W D}$ results from the following considerations. When material starts accumulating on the WD surface, its temperature is lower than the core temperature and also lower than the ignition temperature (otherwise hydrogen would ignite immediately and quietly, rather than explosively under degenerate conditions). As the material becomes 
compressed, it releases gravitational energy, which is absorbed, in part, by the accreted layer, while in part it is conducted into the core. If the absorption of heat is sufficiently effective to raise the temperature of the hydrogen-rich material then, eventually, the temperature at the bottom of the hydrogen-rich layer will become high enough for hydrogen to ignite. The nuclear luminosity, low at first, will soon become the dominant energy source. Therefore, the restrictive condition for a nova outburst to occur is that compressional heating be sufficiently effective in order to raise the temperature to the ignition value of roughly $15 \times 10^{6} \mathrm{~K}$ required by the CNO cycle. The competition between compressional heating power and the rate of heat conduction can only be decided by solving the energy equation. Although it is reasonable to assume that accumulation of matter at a high rate and/or a massive WD will tip the scale in favor of heating, an accurate analytical result is difficult to obtain; it would require too many simplifying assumptions and approximations. We therefore resort to numerical modeling in order to obtain an estimate on the limiting temperature.

A few additional models, beyond the grid described in Section 2 were calculated for WDs cooler than $10^{7} \mathrm{~K}$; indeed very old WDs in binary systems may reach temperatures below $10^{7} \mathrm{~K}$ (Nelson et al. 2004). For high accretion rates $\left(\dot{M}=10^{-9} M_{\odot} \mathrm{yr}^{-1}\right)$, we obtained nova outbursts even for $T_{W D}=3 \times 10^{6} \mathrm{~K}$ and the results were very similar to those obtained for $T_{W D}=10^{7} \mathrm{~K}$. At the other $\dot{M}$ end, adopting $\dot{M}=10^{-12} M_{\odot} \mathrm{yr}^{-1}$ and $M_{W D}=1 M_{\odot}$, we did not obtain an outburst for $T_{W D} / 10^{6} \mathrm{~K}=5,7$ and 8; the accreted material cooled continuously. However, for $T_{W D}=9 \times 10^{6} \mathrm{~K}$ a regular nova outburst was obtained. Thus the lower limit for $T_{W D}$ is strongly dependent on $\dot{M}$, as expected, and in agreement with the conclusions of Schwartzman et al. (1994). In the case of a very cold WD, heating is impeded by heat conduction into the hydrogen-depleted deep core and may be altogether suppressed. However, the impediment is less severe at high accretion rates, due to the rapid supply of accretion energy.

We also checked the effect of $M_{W D}$ : taking $T_{W D} / 10^{6} \mathrm{~K}=8$ (no nova for $M_{W D}=1 M_{\odot}$ ), but increasing the WD mass to $1.25 M_{\odot}$, we did obtain a nova outburst. In this case we found the lower limit to be below $8 \times 10^{6} \mathrm{~K}$ but above $7 \times 10^{6} \mathrm{~K}$. Thus, a lower limit to the WD core temperature exists for any combination of WD mass and accretion rate, but for high accretion rates this limit is so low that it requires more than the age of the universe for a cooling WD to reach it. Hence the constraint on $T_{W D}$ becomes significant only in the case of low accretion rates.

Finally, since it is not clear that erupting WDs may cool down to the theoretical lower limit, and since between the lower limit and $10^{7} \mathrm{~K}$ the results are not highly sensitive to the precise value of $T_{W D}$ (cf. Schwartzman et al. (1994)), we chose $10^{7} \mathrm{~K}$ as the lowest $T_{W D}$ value in the present study, as in Paper I. However, accretion on very cold WDs will be further 
investigated in a future paper, since it is this part of the parameter space that appears likely to shed light on the most peculiar observed novae, showing very high luminosities, relatively low expansion velocities and, apparently, large ejected masses.

\subsection{The full parameter space}

The three surfaces obtained so far and displayed in Fig. 4 describe a restricted, tubeshaped region within the 3D parameter space where conditions for nova outbursts are expected to be satisfied. The additional constraints imposed by the electron degeneracy and mainly concerning the WD temperature determine the ends of the tube.

Hence there appears to be a confined volume of parameter space, where conditions for classical nova outbursts are satisfied. Plotted on top of these surfaces are all of the parameter combination positions that produced "well-behaved" mass-ejecting nova outbursts in our runs (including the three runs for the lowest WD mass of $0.40 M_{\odot}$ and two runs for WD temperatures lower than $10^{7} \mathrm{~K}$ as analyzed in the previous section). There are six "mass ejecting" parameter combinations that lie on the boundaries of the restricting volume, denoted in Fig. 4 by black circles, while the other 75 combinations lie well inside the volume, and are denoted by blue asterisks. As we have explained earlier, the confining volume we have constructed emphasizes the existence of limitations (on each parameter range) and also a fairly clear shape, but one that should be understood as qualitative. Positions of massejecting nova parameter combinations of the numerical calculations may lie on the verge or even slightly outside that volume, since construction of the different surfaces involves many constants, coefficients and approximations, influencing the exact values obtained. It would have been very instructive were we also able to place on this graph the positions of (parameter combinations of) observed novae. Unfortunately, the estimation of all three parameters for any observed eruption is still problematic and involves too many uncertainties. A unique estimation of the three basic parameters that lead to a set of observed characteristics is an ambitious aim yet to be achieved; hopefully it will be, with the help of studies such as the present one.

\section{SUMMARY AND CONCLUSIONS}

In the framework of this study we have extended the 3D parameter grid of multicycle nova evolution to cover combinations with low accretion rates. The main results of this study may be summarized as follows: 
1. The entire range of observed nova characteristics is thoroughly covered by the complete grid of models. Even exceptional observed values such as outburst amplitude of over 19 magnitudes and very high $Z$ are covered by the new grid of results. The majority of the calculated maximum values for the various characteristics is obtained for the lower- $\dot{M}$ runs, down to the lowest accretion rate value of $5 \times 10^{-13} M_{\odot} \mathrm{yr}^{-1}$.

2. There has been a problem deriving ejected mass values as high as those deduced observationally. Moreover, a strong case has been made that much of the ejecta from each nova is missed by observers (Ferland 1998). We have managed to produce a notably high $m_{e j}$ value of $6.6 \times 10^{-4} M_{\odot}$, which is almost a factor 3 greater than the maximum value obtained in the original grid (Paper I). This high $m_{e j}$ was produced for a low $M_{W D}$ at low $\dot{M}$ (combination 065.10.12). The former maximum $m_{e j}$ was obtained for the same $\left[M_{W D}, T_{W D}\right]$ combination but with $\dot{M}$ of $10^{-10} M_{\odot} \mathrm{yr}^{-1}$.

3. For the new runs of lower $\dot{M}$ values we obtain maximum outburst luminosities surpassing the Eddington luminosities (calculated for electron scattering opacities) by factors of up to a few tens, for the whole range of WD masses. The highest super-Eddington luminosities (with correspondingly highest derived outburst amplitudes) are obtained for fast to very-fast novae.

4. We predict the existence of remarkably small amplitude novae with decline times ranging from a week to over a year.

5. In previous studies, as well as the current one, we have seen that by extending the parameter space new features emerge. That raised the question whether the parameter space is at all limited, and if so, what are the exact limits on the three basic independent parameters? Using both models and analytic relations, we derived a confining volume within the three dimensional parameter space, where conditions for nova outbursts are satisfied. Thus the parameter space is indeed limited. As the new grid of models now covers the entire parameter space (assuming WDs composed of CO — but see below), it should enable us to provide explanations for most (all?) novae, including the more peculiar objects among them. This, however, will be the subject of a later study. Of special significance is the existence of a critical accretion rate $\dot{M}_{c r i t}\left(M_{W D}, T_{W D}\right)$ below which conditions are not sufficient to trigger a TNR. The critical mass transfer rate is indeed very low, but seems to be relevant for old close interacting binaries with very low mass companions. For cases like these, the determination of limits is important.

6. Although this study covers the entire range of WD masses, practically up to the Chandrasekhar limit, the assumed composition of the WD core is $\mathrm{C}$ and $\mathrm{O}$ (in equal mass fractions) for all models. At first glance, this might appear unrealistic; very massive WDs are 
believed to emerge after the carbon burning stage in the evolution of a star, and they should be composed of oxygen, neon and magnesium (Gutierrez et al. 1996). Although the mass fractions of these elements and their dependence on the WD mass are still uncertain issues, clearly such WDs should not contain carbon. On the other hand, our grid of models produces results that cover the entire range of observed characteristics (ignoring the breakdown of the total heavy element mass fraction $Z_{e j}$ ). This seems to indicate that they are more widely applicable than imposed by the assumption on the WD composition. In fact, the mechanism of nova eruptions revolves around thermonuclear instability under conditions of electron degeneracy. Electron degeneracy, in turn, is determined by $\mu_{e}=<A / Z>$, which assumes the same value for helium and carbon burning products. In addition, the CNO cycle is not sensitive to the initial CNO breakdown. Consequently, we should not expect significant differences in the outburst characteristics of WDs that differ only in composition, except for the breakdown of $Z_{e j}$. In order to test this expectation we have repeated the calculations for two illustrative models after replacing the carbon in the WD core by neon. The results are summarized in Table 6 and indeed they confirm our prediction. Thus it is not surprising that the entire range of nova characteristics is reproduced by our $\mathrm{CO}$ models and the results should be applicable to novae in general.

7. An intriguing issue related to the investigation of novae is the question of the ultimate fate of the WD. Will it be losing or gaining mass after multiple successive cycles? Will it be able to reach $M_{C h}$, thus acting as a possible SNIa precursor, or not? The ratio $m_{e j} / m_{a c c}$ is shown in Fig. 6 for all parameter combinations. We note that it falls below unity only in a small region of the parameter space, thus strongly reducing the possibility of SNIa to result from accreting WDs. Nevertheless, this region does lead continuously from low mass to the Chandrasekhar limit provided the accretion rate remains very high all along. Therefore, it is possible, at least in principle, for a WD to grow by accretion up to $M_{C h}$. In order to further investigate this problem we need to consider the dynamic evolution of the binary system that determines the evolution of mass transfer rate. Given the accretion rate as a function of time $\dot{M}_{a c c}(t)$, which changes with binary separation and masses, we can calculate the change of $M_{W D}$ with time,

$$
M_{W D}(t)=\int\left[\dot{M}_{a c c}(t)-\dot{M}_{e j}\left(\dot{M}_{a c c}(t), M_{W D}(t)\right)\right] d t
$$

where

$$
\dot{M}_{e j}\left(\dot{M}_{a c c}(t), M_{W D}(t)\right)=m_{e j} / P_{r e c}
$$

is obtained by interpolation on the grid. An example is shown in Fig. 7, based on an evolving accretion rate kindly supplied by Jarrod Hurley, for a binary system of initial masses $0.6 M_{\odot}$ (secondary) and $0.9 M_{\odot}$ (primary). 
8. Finally, in a similar manner, the evolving luminosity of an accreting WD may be constructed as a quasi-periodic function of the variables $L_{a c c}, L_{\max }, t_{3, b o l}$ and $P_{r e c}$ supplied by the grid, all of which change with time as $M_{W D}$ and $\dot{M}_{a c c}$ change. Thus, in future, an important use of the nova grid will be the parameterization of nova evolution for long term modeling of large stellar systems.

We thank Jarrod Hurley for providing us with his results on the genesis of low-mass white

dwarfs in close binaries (the models of Table 1 and Fig. 7). We also thank an anonymous referee for valuable comments and suggestions.

\section{REFERENCES}

Althaus, L. G. et al. 2004, MNRAS, 347, 125

Bond, H. E. et al. 2003, Nature, 422, 455

Duerbeck, H. W. 1987, Space Sci. Rev., 45, 1

Ferland, G. J. 1998, ASP Conf Ser. 137,165

Fujimoto, M. Y. 1982, ApJ, 257, 752

Hamada, T. \& Salpeter, E. E. 1961, ApJ, 134, 683

Gutierrez, J., Garcia-Berro, E., Iben, I. Jr., Isern, J., Labay, J., \& Canal, R. 1996, ApJ, 459, 701

Hurley, J. \& Shara, M. M. 2003, ApJ, 589, 179

Kutter, G. S. \& Sparks, W. M. 1987, ApJ, 321, 386

Marsh, T. R., Dhillon, V. S., \& Duck, S. R. 1995, MNRAS, 275, 828

MacDonald, J. 1983, ApJ, 267, 732

Mestel, L. 1952, MNRAS, 112, 583

Mould, J. et al. 2000, ApJ, 353, L35

Nauenberg, M. 1972, ApJ, 175, 417

Nelson, L. A., MacCannell, K. A., \& Dubeau, E. 2004, ApJ, 602, 938 
Payne-Gaposchkin, C. 1957, The Galactic Novae (Amsterdam:North Holland)

Prialnik, D. 1995, in Cataclysmic Variables, A. Bianchini, M. Della Valle, M. Orio, eds., (The Netherlands: Kluwer), p. 217.

Prialnik, D. and Kovetz, A. 1985, ApJ, 291, 812

Prialnik, D. and Kovetz, A. 1995, ApJ, 445, 789

Prialnik, D., Shara, M. \& Shaviv, G. 1978, A\&A, 62, 339

Regev, O. \& Shara, M. M. 1989, ApJ, 340, 1006

Schwartzman, E., Kovetz, A. \& Prialnik, D. 1994, MNRAS, 269, 323

Shara, M. M., Prialnik, D. \& Shaviv, G. 1977, A\&A, 61, 363

Shara, M. M., Prialnik, D. \& Shaviv, G. 1980, ApJ, 239, 586

Starrfield, S., Sparks, W. \& Shaviv, G. 1987, ApJ, 321, L51

Starrfield, S., Sparks, W. \& Truran, J. 1972, ApJ, 176, 169

Starrfield, S., Sparks, W. \& Truran, J. 1976, ApJ, 208, 819

This preprint was prepared with the AAS LATEX macros v5.2. 
Table 1. Example of Binary Evolution Sequence - Creation of a Low-Mass CO WD

\begin{tabular}{rccrrrrrl}
\hline \hline Time (Myr) & M1 & M2 & \multicolumn{2}{c}{ Types* } & Sep. (AU) & R1/RL1 & R2/RL2 & \\
\hline 0.000 & 2.809 & 0.196 & MS & MS & 110.363 & 0.03 & 0.01 & initial \\
450.482 & 2.809 & 0.196 & HG & MS & 110.363 & 0.07 & 0.01 & ev change \\
453.303 & 2.809 & 0.196 & GB & MS & 110.408 & 0.19 & 0.01 & ev change \\
456.049 & 2.808 & 0.196 & GB & MS & 49.259 & 1.01 & 0.03 & begin RLOF \\
456.049 & 0.414 & 0.196 & HeMS & MS & 0.854 & 1.01 & 0.03 & common env \\
758.047 & 0.413 & 0.196 & HeGB & MS & 0.782 & 0.25 & 0.92 & ev change \\
786.929 & 0.412 & 0.196 & COWD & MS & 0.765 & 0.05 & 0.94 & ev change \\
1010.000 & 0.412 & 0.196 & COWD & MS & 0.718 & 0.05 & 1.01 & begin RLOF* \\
12000.000 & 0.412 & 0.040 & COWD & MS & 0.841 & 0.03 & 1.06 & max time \\
\hline
\end{tabular}

${ }^{*} \mathrm{MS}=$ Main Sequence, HG = Hertzsprung Gap, GB = Giant Branch.

${ }^{* *}$ From this stage onwards CV behavior - continuous mass-loss from secondary to binary with periodic mass ejections. $(\mathrm{RLOF}=$ Roche-lobe overflow) 
Table 2. Complete Grid Results - Characteristics of The Nova Envelope

\begin{tabular}{|c|c|c|c|c|c|c|c|c|c|}
\hline \multicolumn{3}{|c|}{ Param. Comb. } & \multicolumn{7}{|c|}{ Nova Envelope Characteristics } \\
\hline $\begin{array}{l}M_{W D} \\
\left(M_{\odot}\right)\end{array}$ & $\begin{array}{c}T_{W D} \\
\left(10^{6} \mathrm{~K}\right)\end{array}$ & $\begin{array}{c}\log \dot{M} \\
\left(M_{\odot} y r^{-1}\right)\end{array}$ & $\begin{array}{l}m_{a c c} \\
\left(M_{\odot}\right)\end{array}$ & $\begin{array}{c}m_{e j} \\
\left(M_{\odot}\right)\end{array}$ & $Y_{\text {env }}$ & $Y_{e j}$ & $Z_{\text {env }}$ & $Z_{e j}$ & $\begin{array}{c}T_{8, \max } \\
\left(10^{8}{ }^{\circ} K\right)\end{array}$ \\
\hline 0.40 & 10 & -9 & $4.12 E-04$ & $4.55 E-04$ & 0.2568 & 0.2579 & 0.1154 & 0.1204 & 0.90 \\
\hline 0.40 & 10 & -10 & $5.62 E-04$ & $6.76 E-04$ & 0.2358 & 0.2364 & 0.1832 & 0.1893 & 0.98 \\
\hline 0.40 & 10 & -11 & $5.87 E-04$ & $6.96 E-04$ & 0.2444 & 0.2322 & 0.1539 & 0.2075 & 0.95 \\
\hline 0.65 & 10 & -6 & $8.35 E-06$ & $0.00 E+00$ & 0.2830 & $\ldots$ & 0.0208 & $\bar{\cdots}$ & 0.81 \\
\hline 0.65 & 10 & -7 & $2.45 E-05$ & $0.00 E+00$ & 0.3434 & $\ldots$ & 0.0203 & $\ldots$ & 1.10 \\
\hline 0.65 & 10 & -8 & $1.01 E-04$ & $1.03 E-04$ & 0.3754 & 0.3865 & 0.0207 & 0.0215 & 1.34 \\
\hline 0.65 & 10 & -9 & $1.61 E-04$ & $1.63 E-04$ & 0.2540 & 0.2650 & 0.1201 & 0.1350 & 1.39 \\
\hline 0.65 & 10 & -10 & $2.55 E-04$ & $2.76 E-04$ & 0.2407 & 0.2498 & 0.1637 & 0.1786 & 1.67 \\
\hline 0.65 & 10 & -11 & $2.58 E-04$ & $2.37 E-04$ & 0.2570 & 0.2538 & 0.1233 & 0.1847 & 1.39 \\
\hline 0.65 & 10 & -12 & $3.94 E-04$ & $6.66 E-04$ & 0.2660 & 0.1799 & 0.0500 & 0.4458 & 1.59 \\
\hline 0.65 & 10 & $-12.3^{*}$ & $5.40 E-04$ & $5.04 E-04$ & 0.2644 & 0.2723 & 0.0504 & 0.0733 & 1.51 \\
\hline 0.65 & 30 & -6 & $8.63 E-06$ & $0.00 E+00$ & 0.2830 & $\bar{\cdots}$ & 0.0208 & $\cdots$ & 0.80 \\
\hline 0.65 & 30 & -7 & $2.54 E-05$ & $0.00 E+00$ & 0.3690 & $\ldots$ & 0.0203 & $\ldots$ & 1.09 \\
\hline 0.65 & 30 & -8 & $1.02 E-04$ & $1.01 E-04$ & 0.3689 & 0.3801 & 0.0208 & 0.0214 & 1.22 \\
\hline 0.65 & 30 & -9 & $1.11 E-04$ & $1.21 E-04$ & 0.2884 & 0.2956 & 0.1037 & 0.1102 & 1.21 \\
\hline 0.65 & 30 & -10 & $9.55 E-05$ & $1.21 E-04$ & 0.2462 & 0.2489 & 0.2247 & 0.2370 & 1.24 \\
\hline 0.65 & 30 & -11 & $5.96 E-05$ & $9.46 E-05$ & 0.1804 & 0.1817 & 0.3767 & 0.3910 & 1.21 \\
\hline 0.65 & 30 & -12 & $4.12 E-05$ & $9.61 E-05$ & 0.1203 & 0.1216 & 0.5961 & 0.6085 & 1.28 \\
\hline 0.65 & 30 & -12.3 & $4.44 E-05$ & $1.07 E-04$ & 0.1149 & 0.1161 & 0.6170 & 0.6300 & 1.32 \\
\hline 0.65 & 50 & -6 & $8.94 E-06$ & $0.00 E+00$ & 0.2860 & $\cdots$ & 0.0206 & $\cdots$ & 0.80 \\
\hline 0.65 & 50 & -7 & $2.66 E-05$ & $0.00 E+00$ & 0.3497 & $\ldots$ & 0.0203 & $\ldots$ & 0.97 \\
\hline 0.65 & 50 & -8 & $1.06 E-04$ & $9.88 E-05$ & 0.3669 & 0.3774 & 0.0207 & 0.0212 & 1.31 \\
\hline 0.65 & 50 & -9 & $7.41 E-05$ & $9.16 E-05$ & 0.2556 & 0.2584 & 0.2098 & 0.2208 & 1.16 \\
\hline 0.65 & 50 & -10 & $5.23 E-05$ & $6.72 E-05$ & 0.2549 & 0.2570 & 0.2442 & 0.2551 & 1.20 \\
\hline 0.65 & 50 & -11 & $3.86 E-05$ & $5.36 E-05$ & 0.2480 & 0.2550 & 0.3100 & 0.3160 & 1.09 \\
\hline 0.65 & 50 & -12 & $4.54 E-05$ & $1.11 E-04$ & 0.1320 & 0.1347 & 0.6049 & 0.6171 & 1.33 \\
\hline 0.65 & 50 & -12.3 & $1.16 E-04$ & $2.61 E-04$ & 0.1288 & 0.1472 & 0.6262 & 0.6191 & 1.60 \\
\hline 1.00 & 10 & -6 & $2.05 E-06$ & $0.00 E+00$ & 0.3260 & $\cdots$ & 0.0204 & $\cdots$ & 1.05 \\
\hline 1.00 & 10 & -7 & $8.96 E-06$ & $7.21 E-06$ & 0.3421 & 0.4187 & 0.0210 & 0.0223 & 1.41 \\
\hline 1.00 & 10 & -8 & $2.06 E-05$ & $2.22 E-05$ & 0.3044 & 0.3314 & 0.0937 & 0.1002 & 1.59 \\
\hline 1.00 & 10 & -9 & $4.66 E-05$ & $5.18 E-05$ & 0.2676 & 0.2969 & 0.1200 & 0.1270 & 1.86 \\
\hline 1.00 & 10 & -10 & $8.40 E-05$ & $9.72 E-05$ & 0.2427 & 0.2744 & 0.1553 & 0.1620 & 2.10 \\
\hline 1.00 & 10 & -11 & $8.72 E-05$ & $1.00 E-04$ & 0.2475 & 0.2730 & 0.1529 & 0.1917 & 2.09 \\
\hline 1.00 & 10 & -12 & $9.28 E-05$ & $1.62 E-04$ & 0.2429 & 0.1901 & 0.0332 & 0.4770 & 2.10 \\
\hline 1.00 & 10 & -12.3 & $1.04 E-04$ & $2.00 E-04$ & 0.1860 & 0.1772 & 0.0136 & 0.5328 & 2.31 \\
\hline 1.00 & 30 & -6 & $2.10 E-06$ & $0.00 E+00$ & 0.3160 & $\cdots$ & 0.0204 & 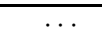 & 1.04 \\
\hline 1.00 & 30 & -7 & $8.74 E-06$ & $5.74 E-06$ & 0.3280 & 0.3535 & 0.0208 & 0.0215 & 1.34 \\
\hline 1.00 & 30 & -8 & $2.03 E-05$ & $2.19 E-05$ & 0.3035 & 0.3300 & 0.0951 & 0.1010 & 1.57 \\
\hline 1.00 & 30 & -9 & $2.70 E-05$ & $3.10 E-05$ & 0.2686 & 0.2940 & 0.1492 & 0.1590 & 1.68 \\
\hline 1.00 & 30 & -10 & $2.10 E-05$ & $2.70 E-05$ & 0.2376 & 0.2587 & 0.2411 & 0.2558 & 1.66 \\
\hline 1.00 & 30 & -11 & $1.15 E-05$ & $1.99 E-05$ & 0.1752 & 0.1884 & 0.4435 & 0.4656 & 1.60 \\
\hline 1.00 & 30 & -12 & $9.72 E-06$ & $2.17 E-05$ & 0.1407 & 0.1665 & 0.5910 & 0.5971 & 1.66 \\
\hline 1.00 & 30 & -12.3 & $3.21 E-05$ & $5.53 E-05$ & 0.1607 & 0.2064 & 0.5588 & 0.5431 & 2.12 \\
\hline 1.00 & 50 & -6 & $2.15 E-06$ & $0.00 E+00$ & 0.3220 & & 0.0203 & & 1.03 \\
\hline
\end{tabular}


Table 2-Continued

\begin{tabular}{|c|c|c|c|c|c|c|c|c|c|}
\hline \multicolumn{3}{|c|}{ Param. Comb. } & \multicolumn{7}{|c|}{ Nova Envelope Characteristics } \\
\hline $\begin{array}{l}M_{W D} \\
\left(M_{\odot}\right)\end{array}$ & $\begin{array}{c}T_{W D} \\
\left(10^{6} \mathrm{~K}\right)\end{array}$ & $\begin{array}{c}\log \dot{M} \\
\left(M_{\odot} y r^{-1}\right)\end{array}$ & $\begin{array}{l}m_{a c c} \\
\left(M_{\odot}\right)\end{array}$ & $\begin{array}{c}m_{e j} \\
\left(M_{\odot}\right)\end{array}$ & $Y_{e n v}$ & $Y_{e j}$ & $Z_{e n v}$ & $Z_{e j}$ & $\begin{array}{c}T_{8, \max } \\
\left(10^{8}{ }^{\circ} \mathrm{K}\right)\end{array}$ \\
\hline 1.00 & 50 & -7 & $8.30 E-06$ & $5.05 E-06$ & 0.3239 & 0.3470 & 0.0207 & 0.0210 & 1.39 \\
\hline 1.00 & 50 & -8 & $2.27 E-05$ & $2.27 E-05$ & 0.3625 & 0.3891 & 0.0227 & 0.0272 & 1.58 \\
\hline 1.00 & 50 & -9 & $1.62 E-05$ & $1.94 E-05$ & 0.2718 & 0.2934 & 0.1836 & 0.1957 & 1.53 \\
\hline 1.00 & 50 & -10 & $1.09 E-05$ & $1.42 E-05$ & 0.2622 & 0.2809 & 0.2486 & 0.2636 & 1.46 \\
\hline 1.00 & 50 & -11 & $7.94 E-06$ & $1.19 E-05$ & 0.2220 & 0.2450 & 0.3710 & 0.3790 & 1.43 \\
\hline 1.00 & 50 & -12 & $2.07 E-05$ & $3.27 E-05$ & 0.2328 & 0.2756 & 0.4323 & 0.4210 & 1.78 \\
\hline 1.00 & 50 & -12.3 & $2.53 E-05$ & $4.69 E-05$ & 0.2274 & 0.2748 & 0.4947 & 0.4764 & 1.96 \\
\hline 1.25 & 10 & -6 & $4.14 E-07$ & $0.00 E+00$ & 0.3184 & $\cdots$ & 0.0204 & $\cdots$ & 1.27 \\
\hline 1.25 & 10 & -7 & $1.92 E-06$ & $1.62 E-06$ & 0.3194 & 0.3744 & 0.0217 & 0.0263 & 1.62 \\
\hline 1.25 & 10 & -8 & $3.67 E-06$ & $3.91 E-06$ & 0.2983 & 0.3637 & 0.0834 & 0.0876 & 1.90 \\
\hline 1.25 & 10 & -9 & $9.27 E-06$ & $1.06 E-05$ & 0.2562 & 0.3336 & 0.1417 & 0.1411 & 2.33 \\
\hline 1.25 & 10 & -10 & $1.91 E-05$ & $2.18 E-05$ & 0.2473 & 0.3221 & 0.1413 & 0.1562 & 2.70 \\
\hline 1.25 & 10 & -11 & $2.97 E-05$ & $3.61 E-05$ & 0.2372 & 0.3155 & 0.1933 & 0.2092 & 3.01 \\
\hline 1.25 & 10 & -12 & $3.22 E-05$ & $5.33 E-05$ & 0.1889 & 0.2485 & 0.3696 & 0.4371 & 3.77 \\
\hline 1.25 & 10 & -12.3 & & & & $\ldots$ & & & \\
\hline 1.25 & 30 & -6 & $3.82 E-07$ & $0.00 E+00$ & 0.3299 & $\cdots$ & 0.0203 & . & 1.23 \\
\hline 1.25 & 30 & -7 & $1.96 E-06$ & $1.86 E-06$ & 0.3837 & 0.4380 & 0.0224 & 0.0273 & 1.67 \\
\hline 1.25 & 30 & -8 & $3.84 E-06$ & $4.16 E-06$ & 0.3027 & 0.3671 & 0.0996 & 0.1037 & 1.92 \\
\hline 1.25 & 30 & -9 & $5.22 E-06$ & $5.86 E-06$ & 0.2730 & 0.3400 & 0.1305 & 0.1359 & 2.05 \\
\hline 1.25 & 30 & -10 & $4.35 E-06$ & $5.55 E-06$ & 0.2423 & 0.3060 & 0.2380 & 0.2480 & 2.05 \\
\hline 1.25 & 30 & -11 & $2.34 E-06$ & $4.26 E-06$ & 0.1676 & 0.2202 & 0.4733 & 0.4931 & 1.99 \\
\hline 1.25 & 30 & -12 & $2.10 E-06$ & $4.25 E-06$ & 0.1421 & 0.2124 & 0.5747 & 0.5725 & 2.01 \\
\hline 1.25 & 30 & -12.3 & $5.79 E-06$ & $9.91 E-06$ & 0.1858 & 0.2708 & 0.5548 & 0.5401 & 2.84 \\
\hline 1.25 & 50 & -6 & $4.16 E-07$ & $0.00 E+00$ & 0.3148 & $\bar{\cdots}$ & 0.0205 & $\cdots$ & 1.26 \\
\hline 1.25 & 50 & -7 & $1.96 E-06$ & $1.78 E-06$ & 0.3374 & 0.4221 & 0.0218 & 0.0269 & 1.67 \\
\hline 1.25 & 50 & -8 & $3.69 E-06$ & $4.01 E-06$ & 0.3026 & 0.3661 & 0.1019 & 0.1069 & 1.89 \\
\hline 1.25 & 50 & -9 & $3.18 E-06$ & $3.58 E-06$ & 0.2803 & 0.3415 & 0.1498 & 0.1583 & 1.86 \\
\hline 1.25 & 50 & -10 & $2.14 E-06$ & $2.78 E-06$ & 0.2584 & 0.3133 & 0.2523 & 0.2663 & 1.78 \\
\hline 1.25 & 50 & -11 & $1.62 E-06$ & $2.51 E-06$ & 0.2210 & 0.2820 & 0.3930 & 0.3970 & 1.76 \\
\hline 1.25 & 50 & -12 & $4.43 E-06$ & $5.95 E-06$ & 0.3387 & 0.4160 & 0.3356 & 0.3264 & 2.12 \\
\hline 1.25 & 50 & -12.3 & $5.57 E-06$ & $1.00 E-05$ & 0.4400 & 0.5262 & 0.3346 & 0.3169 & 2.46 \\
\hline 1.40 & 10 & -6 & $1.81 E-08$ & $0.00 E+00$ & 0.3372 & $\cdots$ & 0.0213 & $\cdots$ & 1.64 \\
\hline 1.40 & 10 & -7 & $7.71 E-08$ & $5.31 E-08$ & 0.3087 & 0.4603 & 0.0218 & 0.0272 & 2.09 \\
\hline 1.40 & 10 & -8 & $1.64 E-07$ & $1.83 E-07$ & 0.2977 & 0.5004 & 0.1277 & 0.1277 & 2.52 \\
\hline 1.40 & 10 & -9 & $4.12 E-07$ & $4.74 E-07$ & 0.2622 & 0.4732 & 0.1536 & 0.1521 & 3.07 \\
\hline 1.40 & 10 & -10 & $5.90 E-07$ & $6.90 E-07$ & 0.2543 & 0.4068 & 0.1591 & 0.2250 & 3.62 \\
\hline 1.40 & 10 & -11 & $2.59 E-06$ & $3.03 E-06$ & 0.2332 & 0.4598 & 0.2077 & 0.1967 & 4.70 \\
\hline 1.40 & 10 & -12 & & & & $\ldots$ & & & \\
\hline 1.40 & 10 & -12.3 & & & & $\ldots$ & & & \\
\hline 1.40 & 30 & -6 & $1.78 E-08$ & $0.00 E+00$ & 0.3466 & $\bar{\cdots}$ & 0.0208 & $\bar{\cdots}$ & 1.62 \\
\hline 1.40 & 30 & -7 & $7.94 E-08$ & $5.54 E-08$ & 0.3073 & 0.4574 & 0.0217 & 0.0271 & 2.10 \\
\hline 1.40 & 30 & -8 & $2.02 E-07$ & $2.02 E-07$ & 0.3600 & 0.5410 & 0.0242 & 0.0339 & 2.51 \\
\hline 1.40 & 30 & -9 & $2.64 E-07$ & $3.02 E-07$ & 0.2817 & 0.4891 & 0.1488 & 0.1497 & 2.81 \\
\hline 1.40 & 30 & -10 & $2.11 E-07$ & $2.68 E-07$ & 0.2509 & 0.4643 & 0.2338 & 0.2325 & 2.76 \\
\hline
\end{tabular}


Table 2-Continued

\begin{tabular}{|c|c|c|c|c|c|c|c|c|c|}
\hline \multirow[b]{2}{*}{$\begin{array}{l}M_{W D} \\
\left(M_{\odot}\right) \\
\end{array}$} & \multirow{2}{*}{\multicolumn{2}{|c|}{$\begin{array}{cc}\text { Param. Comb. } \\
T_{W D} \quad \log \dot{M} \\
\left(10^{6} K\right) & \left(M_{\odot} y r^{-1}\right) \\
\end{array}$}} & \multirow[b]{2}{*}{$\begin{array}{l}m_{a c c} \\
\left(M_{\odot}\right)\end{array}$} & \multicolumn{4}{|c|}{ Nova Envelope Characteristics } & \multirow[b]{2}{*}{$Z_{e j}$} & \multirow[b]{2}{*}{$\begin{array}{r}T_{8, \max } \\
\left(10^{8}{ }^{\circ} \mathrm{K}\right.\end{array}$} \\
\hline & & & & $\begin{array}{c}m_{e j} \\
\left(M_{\odot}\right)\end{array}$ & $Y_{e n v}$ & $Y_{e j}$ & $Z_{e n v}$ & & \\
\hline 1.40 & 30 & -11 & $1.28 E-07$ & $2.09 E-07$ & 0.1968 & 0.3986 & 0.4235 & 0.4325 & 2.65 \\
\hline 1.40 & 30 & -12 & $9.30 E-08$ & $1.94 E-07$ & 0.1189 & 0.3213 & 0.6100 & 0.6179 & 2.66 \\
\hline 1.40 & 30 & -12.3 & & & & $\ldots$ & & & \\
\hline 1.40 & 50 & -6 & $1.80 E-08$ & $0.00 E+00$ & 0.3354 & $\cdots$ & 0.0214 & & 1.62 \\
\hline 1.40 & 50 & -7 & $8.09 E-08$ & $5.69 E-08$ & 0.3089 & 0.4772 & 0.0218 & 0.0277 & 2.07 \\
\hline 1.40 & 50 & -8 & $2.02 E-07$ & $2.02 E-07$ & 0.3600 & 0.5385 & 0.0241 & 0.0336 & 2.48 \\
\hline 1.40 & 50 & -9 & $1.90 E-07$ & $2.18 E-07$ & 0.2927 & 0.4952 & 0.1535 & 0.1541 & 2.60 \\
\hline 1.40 & 50 & -10 & $1.21 E-07$ & $1.64 E-07$ & 0.2608 & 0.4678 & 0.2848 & 0.2838 & 2.48 \\
\hline 1.40 & 50 & -11 & $6.83 E-08$ & $1.25 E-07$ & 0.2220 & 0.4230 & 0.4750 & 0.4730 & 2.40 \\
\hline 1.40 & 50 & -12 & $2.44 E-07$ & $3.15 E-07$ & 0.3772 & 0.6059 & 0.3348 & 0.3202 & 4.20 \\
\hline 1.40 & 50 & -12.3 & & & & $\ldots$ & & & \\
\hline
\end{tabular}

${ }^{*} \log \dot{M}$ of -12.3 stands for $\dot{M}=5 \times 10^{-13}$ 
Table 3. Complete Grid Results - Characteristics of The Outburst

\begin{tabular}{|c|c|c|c|c|c|c|c|c|c|}
\hline \multicolumn{3}{|c|}{ Param. Comb. } & \multicolumn{7}{|c|}{ Outburst Characteristics } \\
\hline$M_{W D}$ & $T_{W D}$ & $\log \dot{M}$ & $\begin{array}{c}v_{\max } \\
\left(k m s^{-1}\right)\end{array}$ & $\begin{array}{c}v_{a v g} \\
\left(k m s^{-1}\right)\end{array}$ & $\begin{array}{c}L_{4, \max } \\
\left(10^{4} L_{\odot}\right)\end{array}$ & $A$ & $\begin{array}{c}t_{3, \text { bol }} \\
(\text { days })\end{array}$ & $\begin{array}{c}t_{m-l} \\
(\text { days })\end{array}$ & $\begin{array}{l}P_{r e c} \\
(y r)\end{array}$ \\
\hline 0.40 & 10 & -9 & 234 & 149 & 7.03 & 15.6 & $1.10 E+04$ & $4.96 E+01$ & $4.12 E+05$ \\
\hline 0.40 & 10 & -10 & 203 & 97 & 11.90 & 18.2 & $8.06 E+03$ & $6.19 E+01$ & $5.62 E+06$ \\
\hline 0.40 & 10 & -11 & 819 & 462 & 23.88 & 21.0 & $5.50 E+04$ & $3.87 E+01$ & $5.87 E+07$ \\
\hline 0.65 & 10 & -6 & 0 & 0 & 1.18 & 4.2 & $2.65 E+04$ & $0.00 E+00$ & $8.35 E+00$ \\
\hline 0.65 & 10 & -7 & 0 & 0 & 1.48 & 6.9 & $5.67 E+04$ & $0.00 E+00$ & $2.45 E+02$ \\
\hline 0.65 & 10 & -8 & 156 & 122 & 1.52 & 9.4 & $2.93 E+04$ & $1.17 E+03$ & $1.01 E+04$ \\
\hline 0.65 & 10 & -9 & 2590 & 2150 & 4.76 & 13.2 & $3.83 E+04$ & $2.64 E+02$ & $1.61 E+05$ \\
\hline 0.65 & 10 & -10 & 4210 & 2650 & 13.70 & 16.8 & $3.23 E+04$ & $1.17 E+02$ & $2.55 E+06$ \\
\hline 0.65 & 10 & -11 & 1300 & 623 & 5.98 & 18.0 & $1.53 E+04$ & $2.76 E+01$ & $2.58 E+07$ \\
\hline 0.65 & 10 & -12 & 682 & 216 & 20.72 & 20.7 & $8.69 E+03$ & $1.10 E+02$ & $3.94 E+08$ \\
\hline 0.65 & 10 & -12.3 & 543 & 230 & 17.34 & 20.9 & $8.22 E+02$ & $6.20 E+02$ & $1.08 E+09$ \\
\hline 0.65 & 30 & -6 & 0 & 0 & 1.17 & 4.1 & $2.82 E+04$ & $0.00 E+00$ & $8.63 E+00$ \\
\hline 0.65 & 30 & -7 & 0 & 0 & 1.49 & 6.9 & $6.01 E+04$ & $0.00 E+00$ & $2.54 E+02$ \\
\hline 0.65 & 30 & -8 & 139 & 125 & 1.58 & 9.5 & $3.22 E+04$ & $1.22 E+03$ & $1.02 E+04$ \\
\hline 0.65 & 30 & -9 & 210 & 156 & 1.65 & 12.0 & $1.27 E+04$ & $6.79 E+02$ & $1.11 E+05$ \\
\hline 0.65 & 30 & -10 & 316 & 195 & 7.96 & 16.2 & $8.38 E+03$ & $4.83 E+02$ & $9.55 E+05$ \\
\hline 0.65 & 30 & -11 & 471 & 189 & 2.16 & 15.2 & $4.38 E+02$ & $3.74 E+01$ & $5.96 E+06$ \\
\hline 0.65 & 30 & -12 & 544 & 268 & 2.72 & 15.4 & $6.08 E+02$ & $2.75 E+01$ & $4.12 E+07$ \\
\hline 0.65 & 30 & -12.3 & 736 & 297 & 2.85 & 15.5 & $5.41 E+02$ & $2.61 E+01$ & $8.89 E+07$ \\
\hline 0.65 & 50 & -6 & 0 & 0 & 1.14 & 4.1 & $2.98 E+04$ & $0.00 E+00$ & $8.94 E+00$ \\
\hline 0.65 & 50 & -7 & 0 & 0 & 1.42 & 6.9 & $6.44 E+04$ & $0.00 E+00$ & $2.66 E+02$ \\
\hline 0.65 & 50 & -8 & 159 & 130 & 1.60 & 9.5 & $4.38 E+04$ & $1.17 E+03$ & $1.06 E+04$ \\
\hline 0.65 & 50 & -9 & 340 & 240 & 1.73 & 12.0 & $7.91 E+03$ & $4.39 E+02$ & $7.41 E+04$ \\
\hline 0.65 & 50 & -10 & 369 & 208 & 1.91 & 13.6 & $7.51 E+03$ & $3.59 E+02$ & $5.22 E+05$ \\
\hline 0.65 & 50 & -11 & 416 & 179 & 2.17 & 13.7 & $6.54 E+03$ & $3.56 E+02$ & $3.86 E+06$ \\
\hline 0.65 & 50 & -12 & 342 & 176 & 2.87 & 16.0 & $5.52 E+03$ & $4.00 E+01$ & $4.54 E+07$ \\
\hline 0.65 & 50 & -12.3 & 552 & 487 & 25.32 & 16.5 & $1.56 E+03$ & $1.69 E+01$ & $2.33 E+08$ \\
\hline 1.00 & 10 & -6 & 0 & 0 & 2.96 & 4.2 & $2.43 E+03$ & $0.00 E+00$ & $2.05 E+00$ \\
\hline 1.00 & 10 & -7 & 265 & 240 & 3.45 & 6.9 & $2.72 E+03$ & $2.10 E+02$ & $8.96 E+01$ \\
\hline 1.00 & 10 & -8 & 351 & 271 & 3.26 & 9.4 & $1.35 E+03$ & $1.27 E+02$ & $2.06 E+03$ \\
\hline 1.00 & 10 & -9 & 525 & 256 & 3.88 & 12.0 & $1.13 E+03$ & $9.35 E+01$ & $4.66 E+04$ \\
\hline 1.00 & 10 & -10 & 1920 & 1180 & 11.30 & 15.7 & $1.28 E+02$ & $3.36 E+01$ & $8.40 E+05$ \\
\hline 1.00 & 10 & -11 & 1250 & 1063 & 6.08 & 17.0 & $2.96 E+03$ & $3.54 E+01$ & $8.72 E+06$ \\
\hline 1.00 & 10 & -12 & 1230 & 292 & 9.80 & 19.1 & $4.19 E+03$ & $5.53 E+01$ & $9.28 E+07$ \\
\hline 1.00 & 10 & -12.3 & 1070 & 295 & 7.46 & 18.7 & $7.14 E+03$ & $5.70 E+01$ & $2.08 E+08$ \\
\hline 1.00 & 30 & -6 & 0 & 0 & 2.96 & 4.2 & $2.53 E+03$ & $0.00 E+00$ & $2.10 E+00$ \\
\hline 1.00 & 30 & -7 & 267 & 237 & 3.26 & 6.9 & $3.35 E+03$ & $2.17 E+02$ & $8.74 E+01$ \\
\hline 1.00 & 30 & -8 & 355 & 274 & 3.28 & 9.4 & $1.32 E+03$ & $1.57 E+02$ & $2.03 E+03$ \\
\hline 1.00 & 30 & -9 & 475 & 324 & 3.61 & 12.0 & $1.02 E+03$ & $1.41 E+02$ & $2.70 E+04$ \\
\hline 1.00 & 30 & -10 & 512 & 344 & 3.55 & 14.4 & $7.08 E+02$ & $1.18 E+02$ & $2.10 E+05$ \\
\hline 1.00 & 30 & -11 & 700 & 446 & 4.17 & 15.3 & $3.93 E+02$ & $1.03 E+01$ & $1.15 E+06$ \\
\hline 1.00 & 30 & -12 & 608 & 414 & 9.14 & 16.5 & $5.28 E+02$ & $1.06 E+01$ & $9.72 E+06$ \\
\hline 1.00 & 30 & -12.3 & 3520 & 3490 & 67.64 & 18.5 & $2.34 E+02$ & $3.82 E+00$ & $6.42 E+07$ \\
\hline 1.00 & 50 & -6 & 0 & 0 & 2.97 & 4.3 & $2.60 E+03$ & $0.00 E+00$ & $2.15 E+00$ \\
\hline
\end{tabular}


Table 3-Continued

\begin{tabular}{|c|c|c|c|c|c|c|c|c|c|}
\hline \multicolumn{3}{|c|}{ Param. Comb. } & \multicolumn{7}{|c|}{ Outburst Characteristics } \\
\hline$M_{W D}$ & $T_{W D}$ & $\log \dot{M}$ & $\begin{array}{c}v_{\max } \\
\left(k m s^{-1}\right)\end{array}$ & $\begin{array}{c}v_{a v g} \\
\left(k m s^{-1}\right)\end{array}$ & $\begin{array}{c}L_{4, \max } \\
\left(10^{4} L_{\odot}\right)\end{array}$ & $A$ & $\begin{array}{c}t_{3, \text { bol }} \\
(\text { days })\end{array}$ & $\begin{array}{c}t_{m-l} \\
(\text { days })\end{array}$ & $\begin{array}{l}P_{r e c} \\
(y r)\end{array}$ \\
\hline 1.00 & 50 & -7 & 300 & 239 & 3.21 & 6.8 & $3.56 E+03$ & $2.53 E+02$ & $8.30 E+01$ \\
\hline 1.00 & 50 & -8 & 360 & 272 & 3.60 & 9.5 & $2.51 E+03$ & $2.40 E+02$ & $2.27 E+03$ \\
\hline 1.00 & 50 & -9 & 400 & 328 & 3.57 & 12.0 & $9.50 E+02$ & $1.43 E+02$ & $1.62 E+04$ \\
\hline 1.00 & 50 & -10 & 384 & 302 & 3.71 & 14.0 & $8.48 E+02$ & $9.14 E+01$ & $1.09 E+05$ \\
\hline 1.00 & 50 & -11 & 680 & 471 & 4.89 & 14.3 & $5.28 E+02$ & $1.22 E+02$ & $7.94 E+05$ \\
\hline 1.00 & 50 & -12 & 736 & 597 & 4.81 & 14.7 & $4.65 E+02$ & $1.16 E+01$ & $2.07 E+07$ \\
\hline 1.00 & 50 & -12.3 & 3170 & 3083 & 89.91 & 18.4 & $2.30 E+02$ & $7.25 E+00$ & $5.05 E+07$ \\
\hline 1.25 & 10 & -6 & 0 & 0 & 4.57 & 4.0 & $3.11 E+02$ & $0.00 E+00$ & $4.14 E-01$ \\
\hline 1.25 & 10 & -7 & 414 & 346 & 4.84 & 6.5 & $2.59 E+02$ & $6.51 E+01$ & $1.92 E+01$ \\
\hline 1.25 & 10 & -8 & 1110 & 568 & 6.38 & 9.3 & $1.31 E+02$ & $2.02 E+01$ & $3.67 E+02$ \\
\hline 1.25 & 10 & -9 & 1480 & 413 & 6.67 & 11.9 & $9.78 E+01$ & $9.56 E+00$ & $9.27 E+03$ \\
\hline 1.25 & 10 & -10 & 2230 & 1940 & 7.14 & 14.5 & $1.38 E+02$ & $2.43 E+01$ & $1.91 E+05$ \\
\hline 1.25 & 10 & -11 & 4240 & 3857 & 69.37 & 19.1 & $4.32 E+02$ & $9.31 E+00$ & $2.97 E+06$ \\
\hline 1.25 & 10 & -12 & 9680 & 8267 & 184.16 & 21.6 & $4.44 E+02$ & $5.86 E+01$ & $3.22 E+07$ \\
\hline 1.25 & 10 & -12.3 & & & & $\cdots$ & & & \\
\hline 1.25 & 30 & -6 & 0 & 0 & 4.52 & 4.0 & $2.46 E+02$ & $0.00 E+00$ & $3.82 E-01$ \\
\hline 1.25 & 30 & -7 & 474 & 373 & 4.77 & 6.5 & $2.36 E+02$ & $6.01 E+01$ & $1.96 E+01$ \\
\hline 1.25 & 30 & -8 & 734 & 531 & 4.78 & 9.0 & $1.62 E+02$ & $3.22 E+01$ & $3.84 E+02$ \\
\hline 1.25 & 30 & -9 & 1030 & 589 & 5.14 & 11.6 & $1.25 E+02$ & $3.14 E+01$ & $5.22 E+03$ \\
\hline 1.25 & 30 & -10 & 1230 & 678 & 6.04 & 14.3 & $1.13 E+02$ & $2.20 E+01$ & $4.35 E+04$ \\
\hline 1.25 & 30 & -11 & 2110 & 1264 & 6.01 & 15.4 & $5.86 E+01$ & $2.05 E+00$ & $2.34 E+05$ \\
\hline 1.25 & 30 & -12 & 2710 & 1696 & 6.60 & 15.8 & $4.61 E+01$ & $2.00 E+00$ & $2.10 E+06$ \\
\hline 1.25 & 30 & -12.3 & 4260 & 3668 & 89.08 & 18.8 & $2.79 E+00$ & $2.20 E+00$ & $1.16 E+07$ \\
\hline 1.25 & 50 & -6 & 0 & 0 & 4.53 & 4.0 & $3.15 E+02$ & $0.00 E+00$ & $4.16 E-01$ \\
\hline 1.25 & 50 & -7 & 436 & 355 & 4.75 & 6.5 & $2.55 E+02$ & $4.75 E+01$ & $1.96 E+01$ \\
\hline 1.25 & 50 & -8 & 807 & 533 & 4.61 & 9.0 & $1.58 E+02$ & $4.30 E+01$ & $3.69 E+02$ \\
\hline 1.25 & 50 & -9 & 689 & 485 & 4.72 & 11.5 & $1.54 E+02$ & $2.47 E+01$ & $3.18 E+03$ \\
\hline 1.25 & 50 & -10 & 921 & 542 & 4.97 & 14.1 & $1.01 E+02$ & $3.04 E+01$ & $2.14 E+04$ \\
\hline 1.25 & 50 & -11 & 889 & 658 & 5.45 & 14.3 & $7.77 E+01$ & $2.59 E+01$ & $1.62 E+05$ \\
\hline 1.25 & 50 & -12 & 1350 & 751 & 6.30 & 14.6 & $6.91 E+01$ & $2.70 E+00$ & $4.43 E+06$ \\
\hline 1.25 & 50 & -12.3 & 3130 & 1675 & 12.25 & 15.9 & $4.45 E+01$ & $2.08 E+00$ & $1.11 E+07$ \\
\hline 1.40 & 10 & -6 & 0 & 0 & 5.92 & 3.4 & $1.08 E+01$ & $0.00 E+00$ & $1.81 E-02$ \\
\hline 1.40 & 10 & -7 & 1410 & 681 & 6.03 & 5.9 & $1.19 E+01$ & $4.13 E+00$ & $7.71 E-01$ \\
\hline 1.40 & 10 & -8 & 3060 & 1160 & 9.27 & 8.8 & $5.02 E+00$ & $1.57 E+00$ & $1.64 E+01$ \\
\hline 1.40 & 10 & -9 & 2850 & 1020 & 9.23 & 11.3 & $4.31 E+00$ & $1.39 E+00$ & $4.12 E+02$ \\
\hline 1.40 & 10 & -10 & 5270 & 2020 & 10.10 & 13.9 & $5.45 E+00$ & $6.78 E-01$ & $5.90 E+03$ \\
\hline 1.40 & 10 & -11 & 4660 & 2817 & 37.54 & 17.5 & $4.71 E+01$ & $2.95 E+00$ & $2.59 E+05$ \\
\hline 1.40 & 10 & -12 & & & & $\cdots$ & & & \\
\hline 1.40 & 10 & -12.3 & & & & $\ldots$ & & & \\
\hline 1.40 & 30 & -6 & 0 & 0 & 5.86 & 3.3 & $1.11 E+01$ & $0.00 E+00$ & $1.78 E-02$ \\
\hline 1.40 & 30 & -7 & 1160 & 723 & 5.99 & 5.9 & $1.19 E+01$ & $3.64 E+00$ & $7.94 E-01$ \\
\hline 1.40 & 30 & -8 & 1760 & 840 & 6.14 & 8.4 & $9.35 E+00$ & $4.63 E+00$ & $2.02 E+01$ \\
\hline 1.40 & 30 & -9 & 3760 & 1800 & 6.98 & 11.0 & $6.17 E+00$ & $1.29 E+00$ & $2.64 E+02$ \\
\hline 1.40 & 30 & -10 & 4490 & 2120 & 7.67 & 13.6 & $4.20 E+00$ & $1.50 E+00$ & $2.11 E+03$ \\
\hline
\end{tabular}


Table 3-Continued

\begin{tabular}{|c|c|c|c|c|c|c|c|c|c|}
\hline \multicolumn{3}{|c|}{ Param. Comb. } & \multicolumn{7}{|c|}{ Outburst Characteristics } \\
\hline$M_{W D}$ & $T_{W D}$ & $\log \dot{M}$ & $\begin{array}{c}v_{\max } \\
\left(k m s^{-1}\right)\end{array}$ & $\begin{array}{c}v_{a v g} \\
\left(k m s^{-1}\right)\end{array}$ & $\begin{array}{c}L_{4, \max } \\
\left(10^{4} L_{\odot}\right)\end{array}$ & $A$ & $\begin{array}{c}t_{3, \text { bol }} \\
(\text { days })\end{array}$ & $\begin{array}{c}t_{m-l} \\
(\text { days })\end{array}$ & $\begin{array}{l}P_{r e c} \\
(y r)\end{array}$ \\
\hline 1.40 & 30 & -11 & 4710 & 2738 & 8.41 & 15.3 & $3.57 E+00$ & $1.87 E-01$ & $1.28 E+04$ \\
\hline 1.40 & 30 & -12 & 3310 & 2312 & 9.52 & 16.1 & $3.05 E+00$ & $1.91 E-01$ & $9.30 E+04$ \\
\hline 1.40 & 30 & -12.3 & & & & $\cdots$ & & & \\
\hline 1.40 & 50 & -6 & 0 & 0 & 5.85 & 3.3 & $1.11 E+01$ & $0.00 E+00$ & $1.80 E-02$ \\
\hline 1.40 & 50 & -7 & 1360 & 812 & 6.01 & 5.8 & $1.25 E+01$ & $4.24 E+00$ & $8.09 E-01$ \\
\hline 1.40 & 50 & -8 & 1540 & 833 & 6.46 & 8.4 & $9.49 E+00$ & $4.80 E+00$ & $2.02 E+01$ \\
\hline 1.40 & 50 & -9 & 3740 & 1580 & 7.18 & 11.1 & $6.03 E+00$ & $1.76 E+00$ & $1.90 E+02$ \\
\hline 1.40 & 50 & -10 & 4350 & 1870 & 7.67 & 13.6 & $4.71 E+00$ & $1.17 E+00$ & $1.21 E+03$ \\
\hline 1.40 & 50 & -11 & 3630 & 1900 & 7.74 & 14.8 & $3.45 E+00$ & $1.67 E+00$ & $6.83 E+03$ \\
\hline 1.40 & 50 & -12 & 3550 & 2570 & 9.31 & 14.9 & $3.81 E+00$ & $3.13 E-01$ & $2.44 E+05$ \\
\hline 1.40 & 50 & -12.3 & & & & $\ldots$ & & & \\
\hline
\end{tabular}

Table 4. Grid Results - Maximum and Minimum Values of Nova Characteristics (for $\left.0.65-1.40 M_{\odot}\right)$

\begin{tabular}{lc|ccc|c|ccc}
\hline \hline & \multirow{2}{*}{ Max } & \multicolumn{3}{c}{ Param. Comb. } & \multicolumn{2}{c}{ Min } & \multicolumn{3}{c}{ Param. Comb. } \\
Characteristic & Value & $M_{W D}$ & $T_{W D}$ & $\log \dot{M}$ & Value & $M_{W D}$ & $T_{W D}$ & log $\dot{M}$ \\
\hline$m_{\text {acc }}$ & $5.40 E-04$ & 0.65 & 10 & -12.3 & $6.83 E-08$ & 1.40 & 50 & -11 \\
$m_{e j}$ & $6.66 E-04$ & 0.65 & 10 & -12 & $5.31 E-08$ & 1.40 & 10 & -7 \\
$Z_{e j}$ & 0.63 & 0.65 & 30 & -12.3 & 0.02 & 1.00 & 50 & -7 \\
$Y_{e j}$ & 0.61 & 1.40 & 50 & -12 & 0.12 & 0.65 & 30 & -12.3 \\
$X_{e j}$ & 0.65 & 0.65 & 10 & -12.3 & 0.06 & 1.40 & 30 & -12 \\
$T_{8, \text { max }}$ & 4.7 & 1.40 & 10 & -11 & 1.1 & 0.65 & 50 & -11 \\
$v_{\text {max }}$ & 5270 & 1.40 & 10 & -10 & 139 & 0.65 & 30 & -8 \\
$v_{\text {avg }}$ & 3860 & 1.25 & 10 & -11 & 122 & 0.65 & 10 & -8 \\
$L_{4, \text { bol,max }}$ & 90 & 1.00 & 50 & -12.3 & 1.5 & 0.65 & 10 & -8 \\
$M_{\text {bol,max }}$ & -10.2 & 1.00 & 50 & -12.3 & -5.7 & 0.65 & 10 & -8 \\
$A$ & 20.9 & 0.65 & 10 & -12.3 & 5.8 & 1.40 & 50 & -7 \\
$t_{3, \text { bol }}$ & 120 yr & 0.65 & 50 & -8 & $3.05 d$ & 1.40 & 30 & -12 \\
$t_{m-l}$ & 67 yr & 0.65 & 10 & -11 & $0.68 d$ & 1.40 & 10 & -10 \\
$P_{\text {rec }}$ & $1.08 E+09$ yr & 0.65 & 10 & -12.3 & $281 d$ & 1.40 & 10 & -7 \\
\hline
\end{tabular}


Table 5. Observed vs. Calculated Nova Characteristics Ranges

\begin{tabular}{lc|cc}
\hline \hline Characteristic & Observed Ranges & Observed Exceptions & Calculated Ranges \\
\hline$M_{\max }$ & -6 to -9 & -10 (V1500 Cyg) & -5.7 to -10.2 \\
$A$ & $7-16$ & 19.3 (V1500 Cyg) & $5.8-20.9$ \\
$t_{3}$ & $4-300$ days & SymN:more & $0.76 d-67 \mathrm{yr}$ \\
$m_{e j}$ & $1-30\left(E-05 M_{\odot}\right)$ & RN:less & $5.3 E-08-6.6 E-04$ \\
$Z_{e j}$ & $0.04-0.41$ & $0.86(\mathrm{~V} 1370 \mathrm{Aql})$ & $0.02-0.63$ \\
$Y_{e j}$ & $0.21-0.48$ & $0.1(\mathrm{~V} 1370 \mathrm{Aql})$ & $0.12-0.60$ \\
$v_{e x p}{ }^{*}$ & $350-2500 \mathrm{~km} \mathrm{~s}^{-1}$ & SymN: $100 \mathrm{~km} \mathrm{~s}^{-1}$ & $122-3860$ \\
\hline
\end{tabular}

*average expansion velocities

Table 6. Ejecta Composition - CO vs. ONe Models

\begin{tabular}{c||cc|cc}
\hline \hline \multicolumn{1}{l||}{} & $1.25,10,-11$ & \multicolumn{2}{c}{$1.40,10,-9$} \\
\multicolumn{1}{c}{} & $C O$ & $O N e$ & $C O$ & $O N e$ \\
\hline$m_{e j}$ & $3.61 E-05$ & $3.67 E-05$ & $4.74 E-07$ & $2.89 E-07$ \\
$y_{e j}$ & 0.3155 & 0.3123 & 0.4732 & 0.5129 \\
$z_{e j}$ & 0.2092 & 0.2179 & 0.1521 & 0.1508 \\
\hline${ }^{*} C^{12}$ & $3.353 E-02$ & $1.970 E-02$ & $2.430 E-02$ & $1.143 E-02$ \\
$C^{13}$ & $3.136 E-02$ & $2.089 E-02$ & $2.485 E-02$ & $8.148 E-03$ \\
$N^{14}$ & $6.545 E-02$ & $3.086 E-02$ & $9.392 E-02$ & $4.706 E-02$ \\
$N^{15}$ & $6.660 E-02$ & $3.321 E-02$ & $4.191 E-03$ & $1.147 E-03$ \\
$O^{16}$ & $1.165 E-02$ & $9.241 E-05$ & $2.092 E-04$ & $1.402 E-04$ \\
$O^{17}$ & $6.157 E-04$ & $7.729 E-05$ & $1.342 E-05$ & $8.500 E-06$ \\
$N e$ & $3.384 E-07$ & $6.579 E-02$ & $1.372 E-07$ & $1.358 E-03$ \\
$N a$ & $3.535 E-09$ & $6.541 E-04$ & $1.047 E-10$ & $1.141 E-06$ \\
$M g$ & $2.861 E-08$ & $9.611 E-03$ & $5.238 E-09$ & $3.640 E-05$ \\
$A l^{26}$ & $2.098 E-08$ & $7.929 E-03$ & $6.355 E-09$ & $4.258 E-05$ \\
$A l^{27}$ & $1.027 E-08$ & $3.137 E-03$ & $1.251 E-09$ & $7.662 E-06$ \\
$\geq S i$ & $1.046 E-07$ & $2.600 E-02$ & $6.087 E-07$ & $8.147 E-02$ \\
\hline
\end{tabular}

*Heavy elements $Z_{e j}$ breakdown 


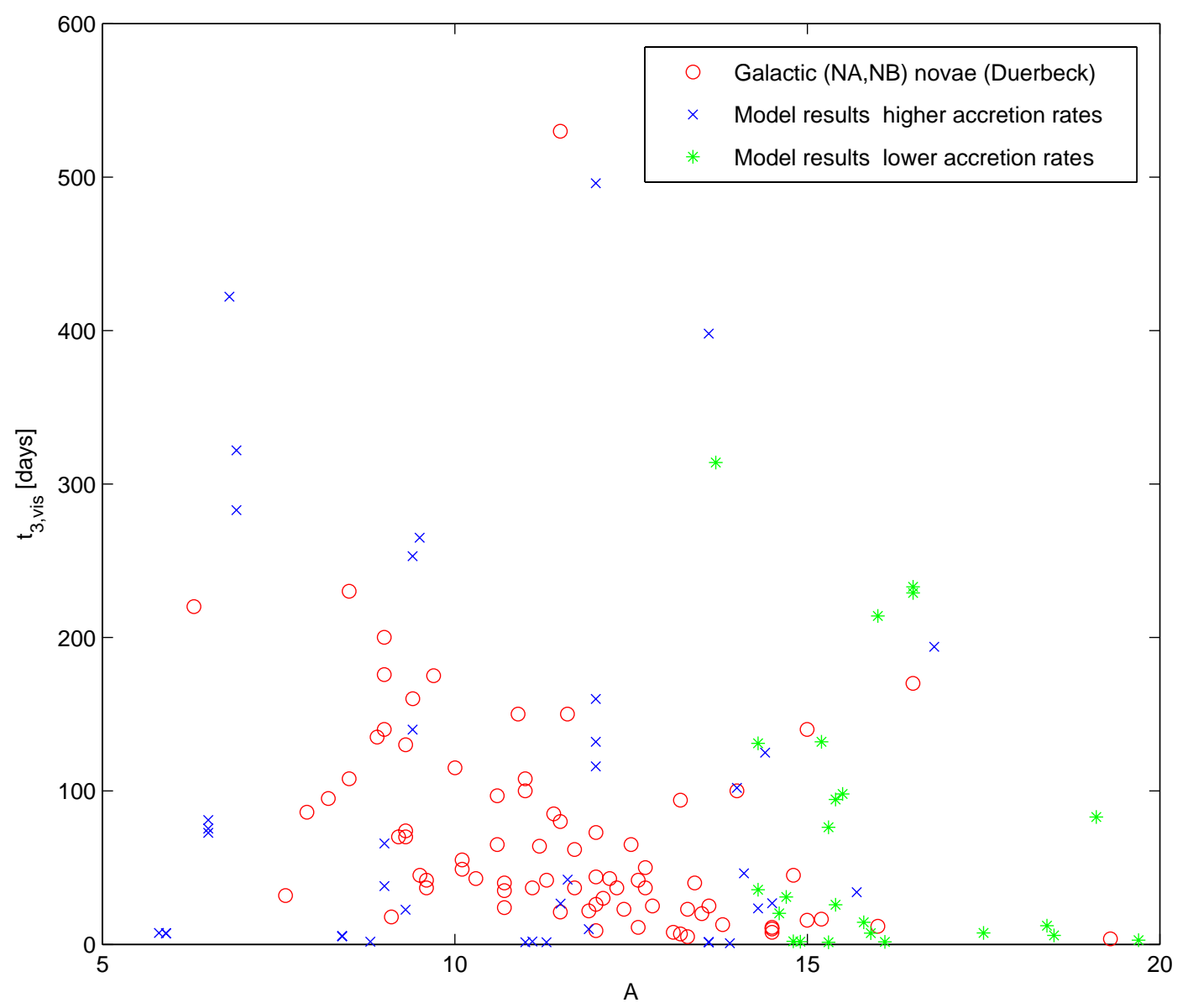

Fig. 1.- Time of decline $t_{3, v i s}$ (order of $t_{m-l}$ ) versus the outburst amplitude $A$ for both observed $N A+N B$ galactic novae (taken from Duerbeck 1987) - circles, and our model results: crosses $-\dot{M} \geqq 10^{-10}$, asterisks $-\dot{M} \leqq 10^{-11}$. 

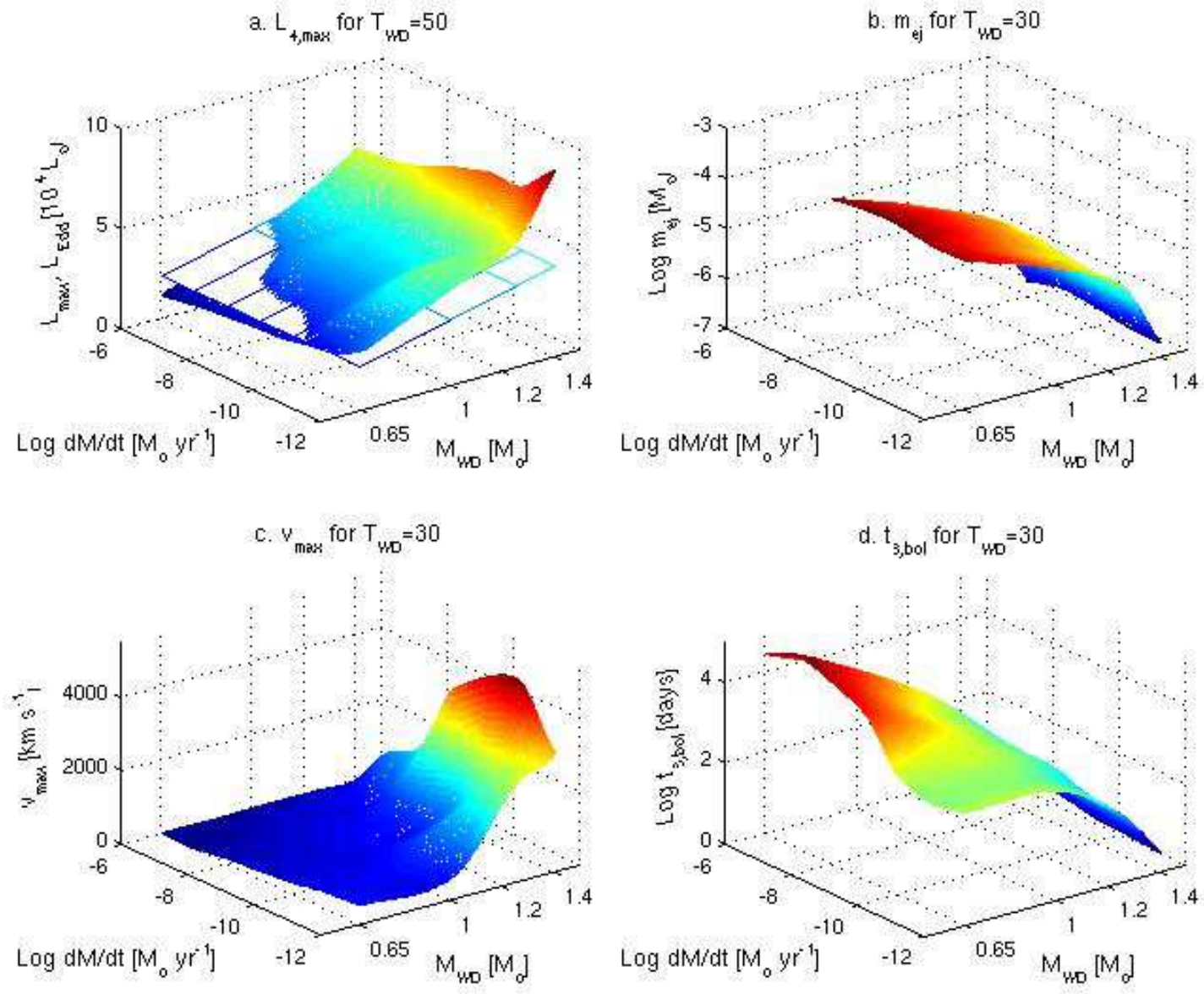

Fig. 2.-3D plots of four major properties out of the complete result grid. Each plot represents the property as function of $\left[\dot{M}, M_{W D}\right]$ for a representative WD temperature (stated in units of $10^{6} \mathrm{~K}$ ). In panel a, the white surface represents the critical Eddington luminosity, emphasizing the domains where the luminosity either surpasses or is lower than $L_{E d d}$. 


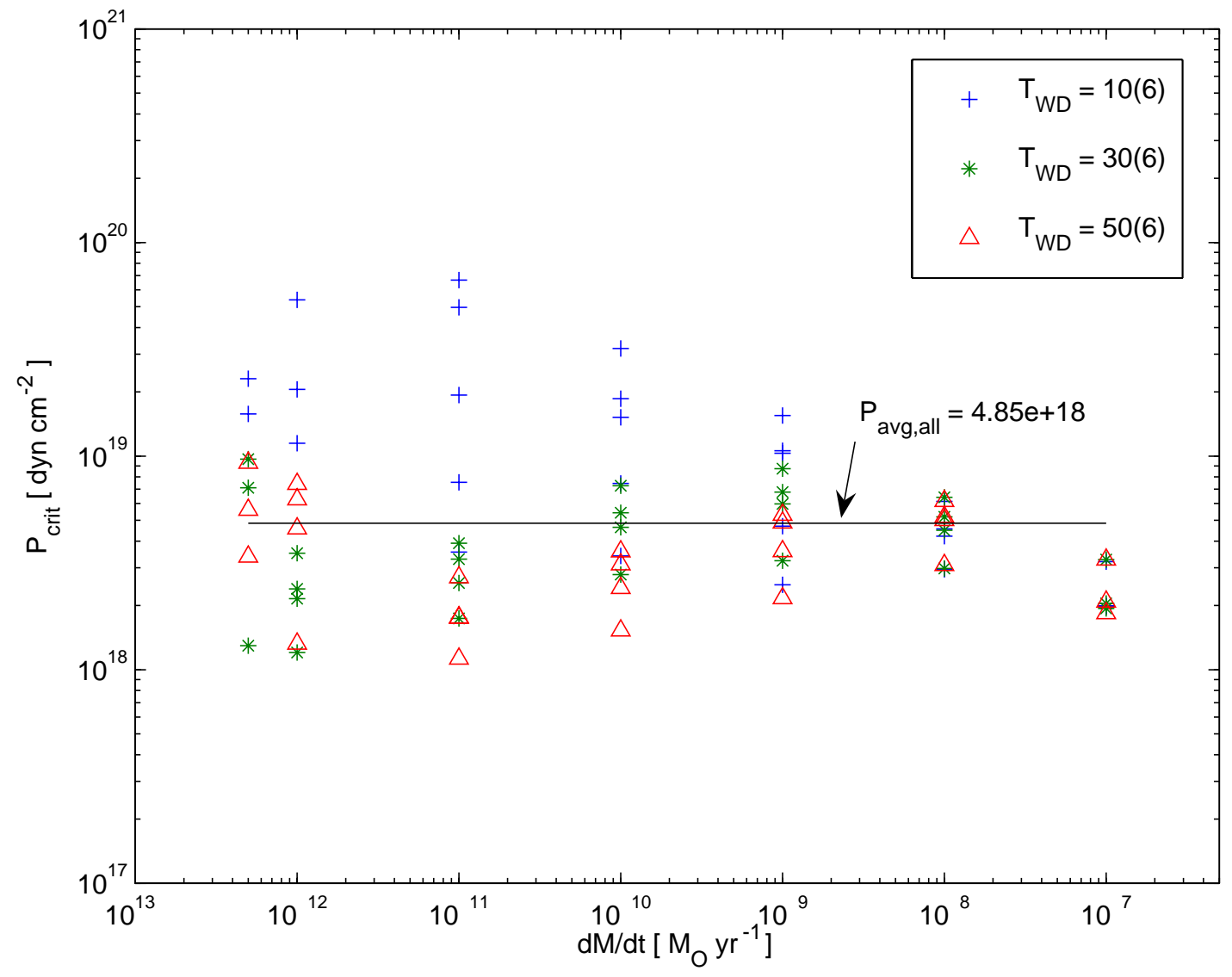

Fig. 3. - Values of the pressure at the base of the envelope prior to the TNR, grouped by the three main $T_{W D}$ values as function of the accretion rate; calculated from grid results (according to eq. 5). 


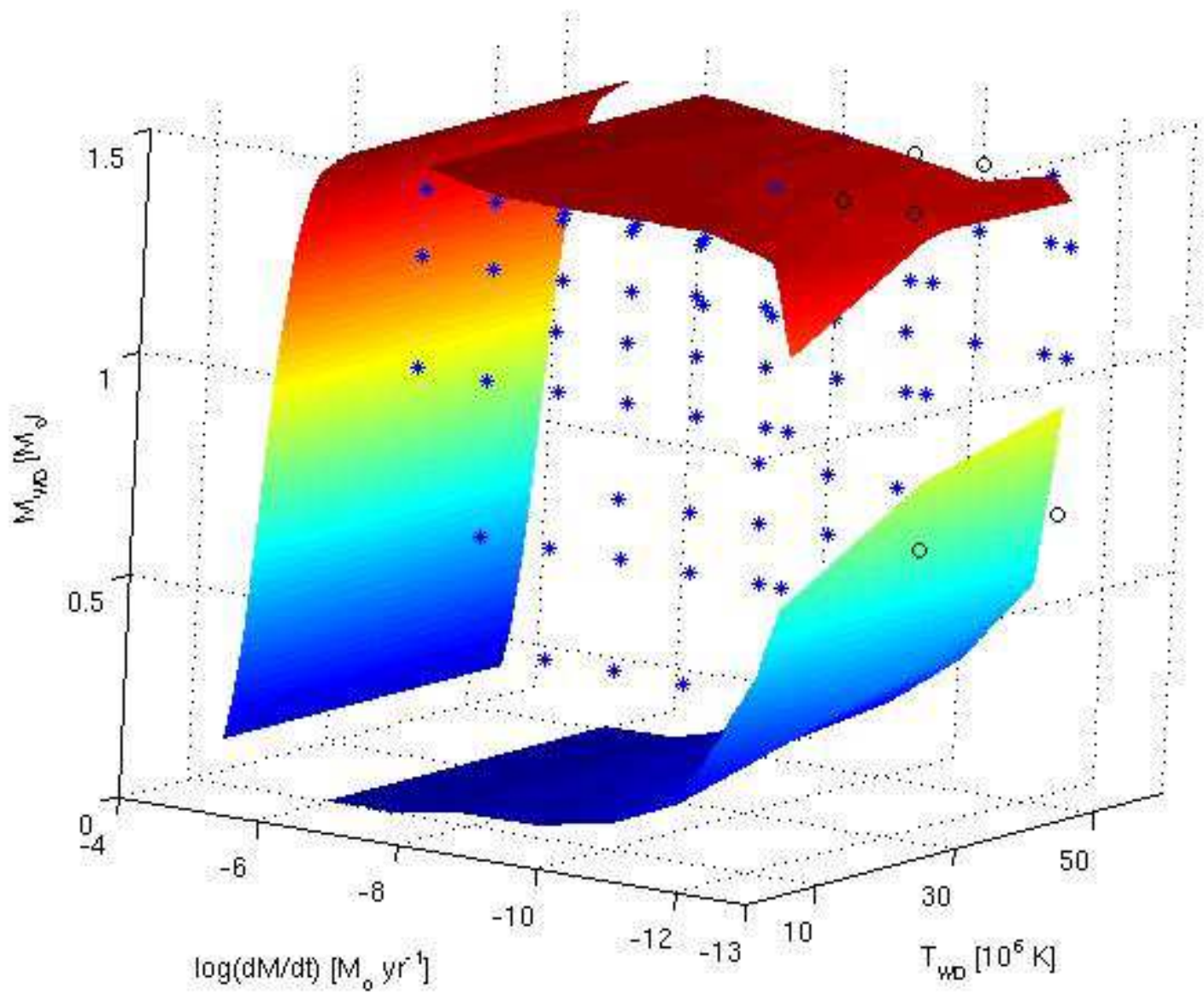

Fig. 4.- The combined restricting surfaces, confining a volume within the 3D parameter space where conditions for nova outbursts are satisfied. The bottom surface corresponds to the heating versus cooling criterion (section 3.1); the top WD mass-limiting surface corresponds to the nuclear versus gravitational energy considerations (section 3.2); the left mass transfer rate-limiting surface relates to the accretion versus Eddington luminosity (section 3.3). Positions of the grid parameter combinations that successfully produced nova eruptions are plotted within the confined volume. 

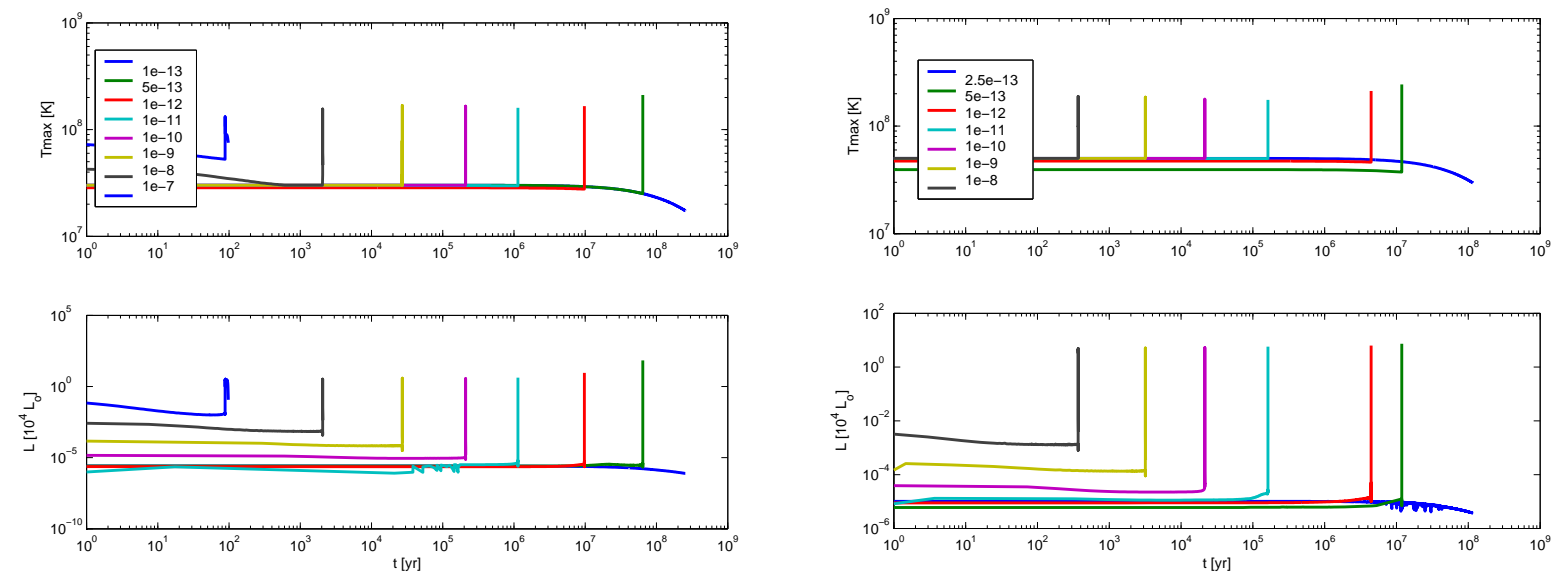

Fig. 5.- Evolution in time of two characteristics for different accretion rates: the maximum temperature (within the burning shells) $T_{\max }$ and the luminosity $L$, for $M_{W D}=$ $1.00 M_{\odot}, T_{W D}=30 \times 10^{6} \mathrm{~K}$ (left), and $M_{W D}=1.25 M_{\odot}, T_{W D}=50 \times 10^{6} \mathrm{~K}$ (right). 

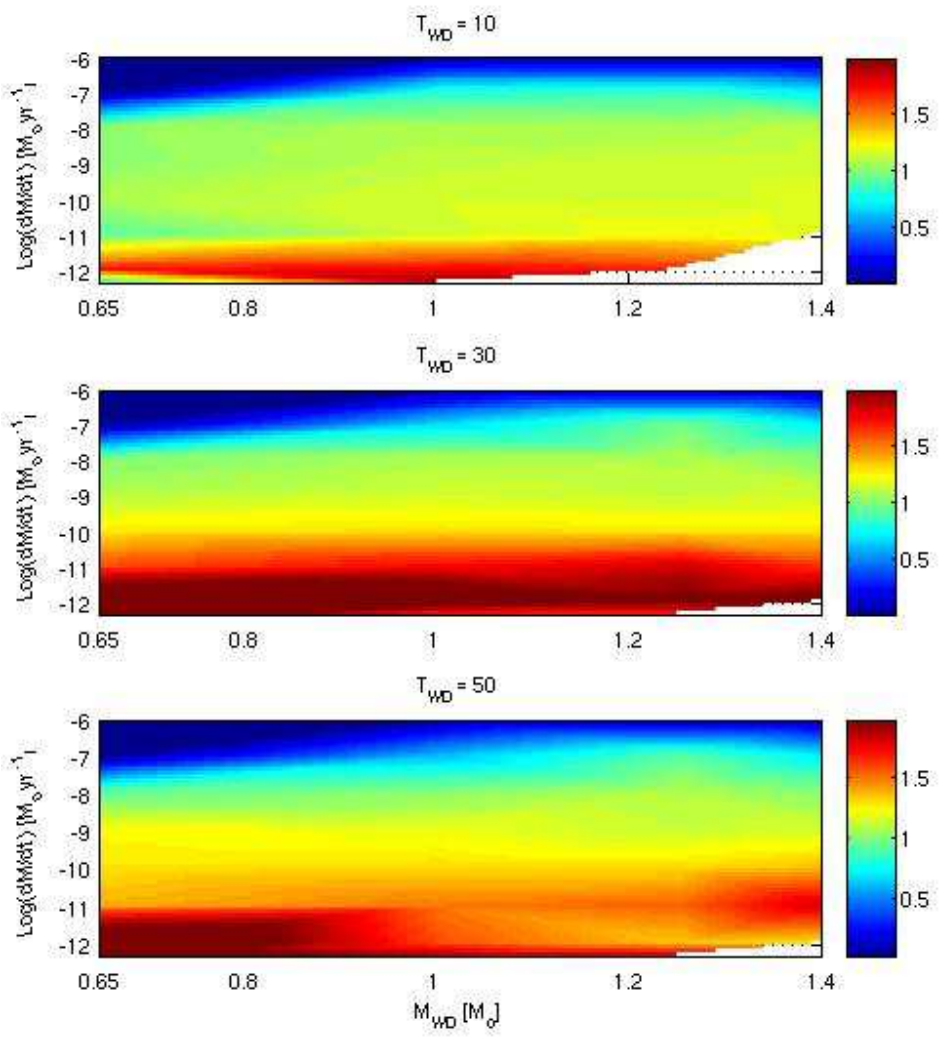

Fig. 6.- Color maps displaying shades that correspond to values of the ratio $m_{e j} / m_{a c c}$ in the $\left[M_{W D}, \log \dot{M}\right]$ plane. A value of 1 corresponds to an unevolving WD mass, below - an increase in $M_{W D}$, and above - a decrease in time of $M_{W D}$. The three panels correspond to the three $T_{W D}$ values - 10,30 and $50\left(10^{6} \mathrm{~K}\right)$, top to bottom. 


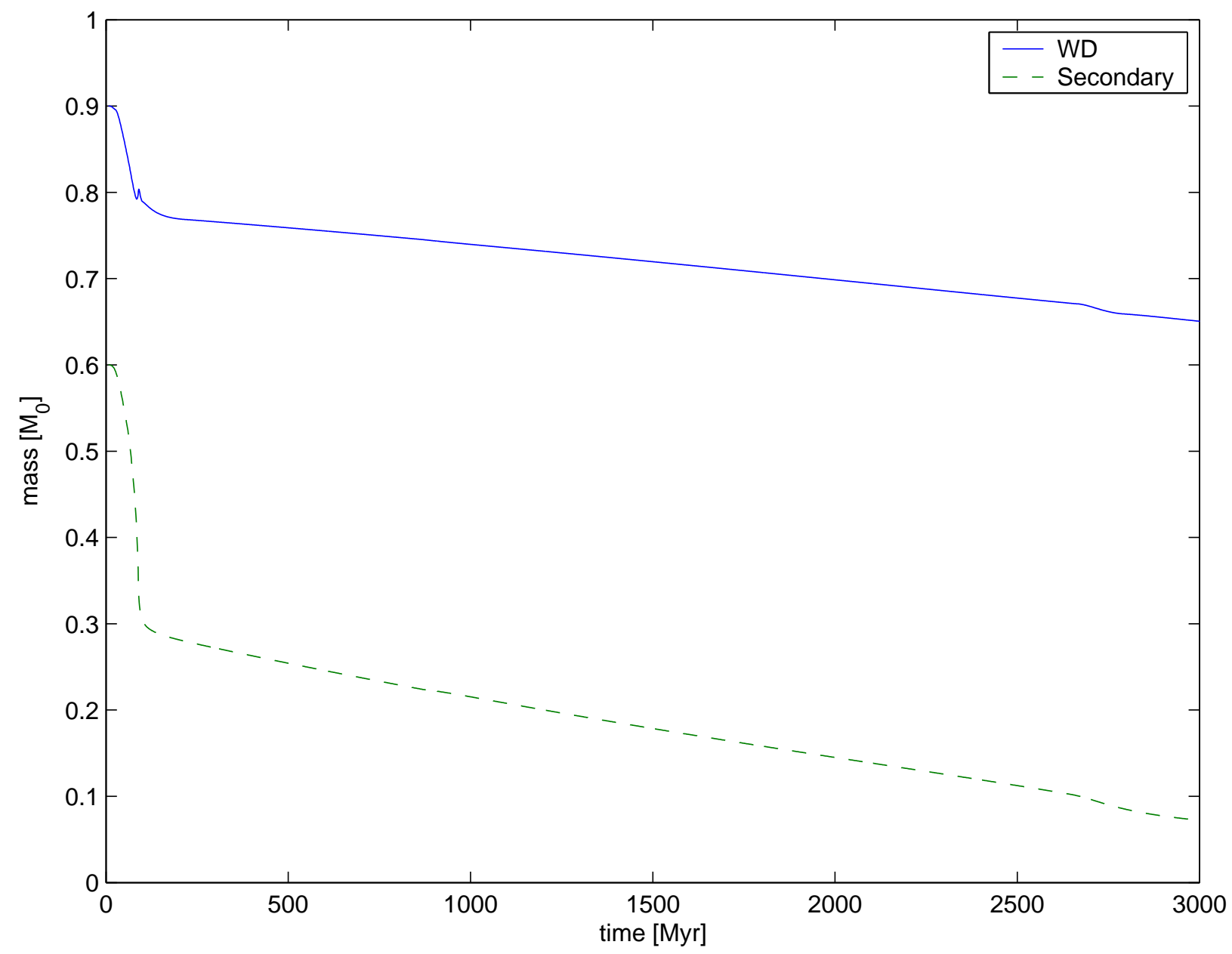

Fig. 7.- An example calculation of $M_{W D}(t)$ based on an evolving $\dot{M}$ for initial masses of 0.6 and $0.9 M_{\odot}$ of the secondary and primary respectively. 